Boletín de la Sociedad Geológica Mexicana

VOLUMEN 62, NÚM. 1, 2010, P. 25-42

\title{
Criterios para la exploración minera mediante microtermometría de inclusiones fluidas
}

\author{
Antoni Camprubí
}

Departamento de Geoquímica, Instituto de Geología, Universidad Nacional Autónoma de México, Ciudad Universitaria, 04510, México D.F., México.

taga@geologia.unam.mx

\section{Resumen}

La microtermometría de inclusiones fluidas es una técnica analítica con multitud de aplicaciones, tanto en estudios académicos como en exploración minera. Esta técnica, sobre una sólida base de estudios petrográficos, permite determinar o estimar (1) las temperaturas de formación de un depósito mineral, (2) la salinidad y contenido en diversos solutos de los fluidos mineralizantes, (3) la evolución temporal y la zonación espacial de la temperatura y la salinidad, (4) la existencia de diversos mecanismos de precipitación mineral, (5) la existencia de pulsos hidrotermales de diversa índole, (6) la presencia de canales de alimentación en un depósito hidrotermal y relacionar la posición de éstos con la distribución de mineralizaciones económicas, (7) la migración de salmueras a través de una cuenca sedimentaria y su relación con la maduración del petróleo o la formación de ciertos tipos de depósitos minerales, (8) la interacción entre fluidos químicamente contrastantes, (9) la posición de la paleosuperficie o del paleonivel freático bajo presión hidrostática, y (10) la tipología o subtipología a la que puede pertenecer una mineralización concreta, así como muchas otras cuestiones de índole metalogenética. De esta forma, esta técnica potencialmente aporta información esencial acerca de la génesis de la mayoría de yacimientos minerales, y puede ser insustituible para guiar o condenar la exploración en un yacimiento o distrito minero.

Palabras clave: inclusiones fluidas, exploración minera, temperatura, salinidad, zonación térmica, depósitos epitermales, skarns, depósitos MVT.

\section{Abstract}

The microthermometry of fluid inclusions is an analytical technique that has numerous applications in both academia and mining exploration. This technique, when based on sound petrographic studies, allows determination or estimation of (1) the formation temperatures of a mineral deposit, (2) the salinity and contents of several solutes in mineralizing fluids, (3) the evolution in time and the spatial variations in temperature and salinity, (4) the occurrence of various mechanisms of mineral deposition, (5) the occurrence of hydrothermal brine pulses, various in nature, (6) the presence of feeder channels in hydrothermal deposits and their relationship with the distribution of economic mineralization, (7) the migration of brines through a sedimentary basin and the association of such phenomena with the maturation of petroleum or the formation of certain types of ore deposits, (8) the interaction between fluids of contrasting chemical composition, (9) the position of paleosurfaces or the paleowater table under hydrostatic pressure, and (10) the type or subtype of ore deposit to which a given deposit may belong, as well as many other metallogenic issues. Consequently, the use of this technique potentially provides key information on the genesis of most ore deposits, and cannot be replaced by other techniques as a guide for mineral exploration or for deciding against exploitation of a mineral deposit or mining district.

Keywords: fluid inclusions, mining exploration, temperature, salinity, thermal zonation, epithermal deposits, skarns, MVT deposits. 


\section{Introducción}

Las inclusiones fluidas son interrupciones o defectos en la estructura cristalina de un mineral que han atrapado algún tipo de fluido durante el crecimiento del mismo. Éstas representan porciones atrapadas de los líquidos, gases y fundidos a partir de los cuales se produjo el crecimiento cristalino. Por ello, las inclusiones fluidas (incluyendo las de fundido) pueden ser usadas, previo análisis petrográfico, para reconstruir las condiciones ambientales del crecimiento cristalino, así como para caracterizar químicamente los fluidos a partir de los que se formaron los minerales que las contienen (e. g. Roedder, 1984; Goldstein y Reynolds, 1994; Van den Kerkhof y Hein, 2001).

La microtermometría de inclusiones fluidas es una técnica analítica que permite determinar ciertas variables físicoquímicas básicas, con aplicación tanto para el campo académico acerca de los fluidos mineralizantes de numerosas tipologías de yacimientos minerales, como para la exploración minera. Además, esta técnica es la base de la aplicación de técnicas analíticas "avanzadas" o trazadoras del origen de los fluidos tales como la geoquímica elemental de halógenos en los solutos, la geoquímica de gases mediante espectrometría de masas cuadrupolar (QMS) o la geoquímica isotópica de gases nobles. Entre las variables de interés que esta técnica permite determinar, las principales son (1) la temperatura de homogeneización, que constituye la temperatura mínima de atrapamiento de las inclusiones fluidas y que, en determinadas circunstancias, equivale a la temperatura de atrapamiento, y (2) la salinidad del fluido, expresada como porcentaje en peso equivalente de $\mathrm{NaCl} y$, en caso de inclusiones con cristales hijos, expresada como porcentaje en peso de $\mathrm{NaCl}, \mathrm{KCl}, \mathrm{MgCl}_{2}$, u otros solutos. Existen otros tipos de geotermómetros y geobarómetros, de tipo paragenético, de química mineral o isotópicos, pero la microtermometría de inclusiones fluidas tiene las ventajas de: no estar sujeta a un mineral concreto o a un grupo restringido de minerales (salvo a los que son mínimamente transparentes), permitir la obtención de temperaturas a microescala, pudiéndose trazar temporalmente la evolución de un fluido mineralizante a escalas muy diversas, ser la única técnica que permite caracterizar la composición en solutos de los fluidos mineralizantes, permitir reconstrucciones difíciles o imposibles de establecer mediante otras técnicas, como isotermas detalladas a escala de depósito, distrito o provincia metalogenética, así como estimaciones de la posición de paleosuperficies o paleoniveles freáticos, y las inclusiones fluidas no son afectadas por procesos de oxidación superficial o meteorización.

Esta es, por lo tanto, una técnica sumamente versátil que ha devenido rutinaria en la investigación académica en el campo de la metalogenia. A pesar de que su uso en la exploración minera ha crecido considerablemente, todavía no constituye una técnica de uso generalizado en dicha actividad. Asimismo, esta técnica adquiere mucha mayor potencia cuando se apoya en estudios petrográficos y mineralógicos detallados (difracción de rayos-X, espectrofotometría de infrarrojo, microscopía electrónica de barrido, microsonda electrónica, etc.). Muchos de estos métodos, incluida la microtermometría de inclusiones fluidas, son relativamente baratos y proporcionan información inmediata, con lo que no resultan onerosos para la exploración minera.

Los datos obtenidos mediante microtermometría de inclusiones fluidas son útiles (y, en algunos casos, insustituibles) para evaluar numerosas cuestiones de orden metalogenético como (1) el rango de temperatura y presión de formación de un yacimiento, (2) la capacidad de transporte de ciertos metales por los fluidos, (3) los mecanismos de precipitación mineral o (4) para acotar las posibles fuentes de los fluidos mineralizantes. Adicionalmente, la distribución espacial de los datos microtermométricos, en conjunto con la distribución de determinados minerales o asociaciones minerales, es particularmente útil para obtener criterios de exploración en un cuerpo mineralizado conocido, o bien en un área no minada en proceso de evaluación. En el presente trabajo se utilizan modelos demostrativos de diversos tipos de yacimientos minerales, como epitermales, skarns, pórfidos metalíferos y depósitos tipo Mississippi Valley, para ejemplificar la explicación.

\section{Condicionantes petrográficos para la microtermo- metría de inclusiones fluidas}

Antes de proceder al análisis microtermométrico de las inclusiones fluidas y después de haber realizado un estudio petrográfico convencional de las asociaciones minerales objeto de estudio, así como de las diversas fases minerales de la sílice (Sander y Black, 1988; Albinson, 1995; Dong et al., 1995; Camprubí y Albinson, 2006), es necesario llevar a cabo un estudio petrográfico de las diversas asociaciones de inclusiones fluidas presentes. La petrografía convencional debe llevar al establecimiento de una secuencia paragenética, con una identificación del tiempo relativo de formación de eventos y micro-eventos (definidos como etapas, sub-etapas, asociaciones y paragénesis minerales). Tal secuencia permite ubicar en un marco temporal los fenómenos geológicos asociados a la precipitación de los minerales que la componen (Figura 1), lo cual incluye el atrapamiento de inclusiones fluidas. La petrografía de inclusiones fluidas asume el mismo esquema operativo, la definición y ordenación relativa de eventos, aunque a microescala. Sin embargo la petrografía de inclusiones fluidas, al enfocarse a texturas y morfologías intracristalinas (no intercristalinas como la petrografía convencional), posee ciertas particularidades. Así, los objetivos básicos de la petrografía de inclusiones fluidas pueden resumirse como determinación de la presencia y las relaciones espaciales de asociaciones o grupos discretos genética, temporal y estadísticamente significativos de 


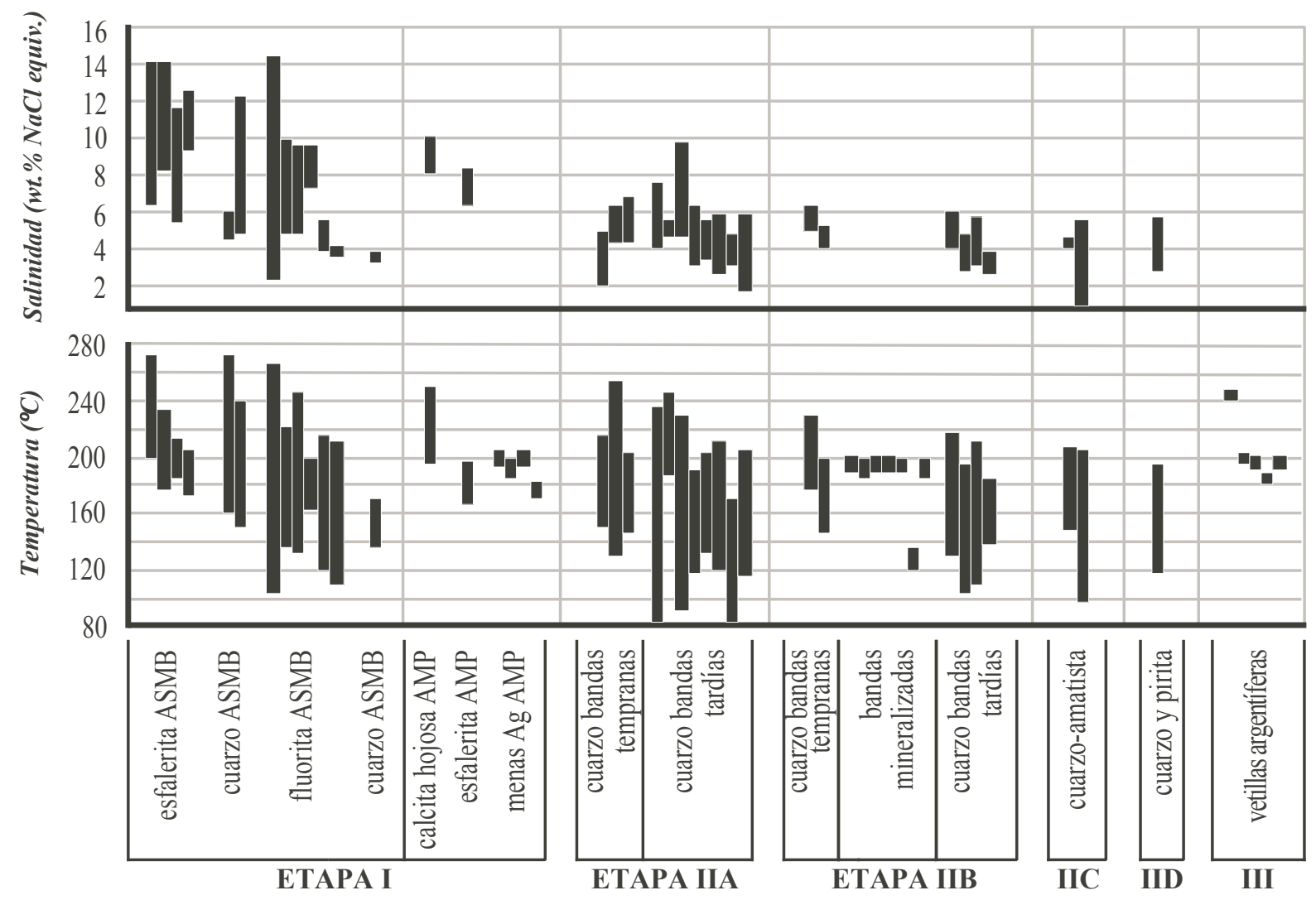

Figura 1. Resumen de las variaciones de salinidad y temperatura (microtermometría de inclusiones fluidas y otros geotermómetros) como rangos de variación completos con el tiempo de los fluidos mineralizantes en el cortejo de vetas epitermales La Guitarra - El Coloso (distrito de Temascaltepec, Estado de México). Los valores se representan de acuerdo con la posición en el tiempo de cada asociación mineral, y de la parte inferior de la veta a la superior, en cada caso. Adaptado de Camprubí et al. (2001b). Clave: ASMB = asociaciones de sulfuros de metales básicos, AMP = asociaciones con minerales de plata.

inclusiones fluidas con los minerales que las contienen. En cualquier caso de estudio, las asociaciones de inclusiones fluidas deben entenderse como las unidades básicas del estudio petrográfico y microtermométrico; determinación de los aspectos morfológicos y geométricos que puedan constituirse en elementos de discusión de los datos microtermométricos, tanto en lo referente a las inclusiones individuales como a las asociaciones de éstas; ello incluye (a) la determinación de los componentes constitutivos de las inclusiones fluidas (fase vapor, líquidos de diversos tipos, vidrio, mineralogía de cristales atrapados e hijos), (b) el grado de relleno, (c) los criterios para la determinación de tipos petrográficos de inclusiones fluidas (primario, secundario y pseudosecundario), y (d) la presencia y naturaleza de modificaciones post-atrapamiento, etc.; determinación de algunos procesos de precipitación mineral, y del tipo de atrapamiento de las inclusiones fluidas cogenéticas, bien sea a partir de un medio homogéneo o de uno heterogéneo (por desmezcla de líquidos, soluciones en ebullición o efervescencia, presencia de sólidos suspendidos y/o existencia de una solución saturada; ver Figura 7 en Van den Kerkhof y Hein, 2001).
Estos aspectos son los que, en último término, condicionan la selección de inclusiones fluidas para microtermometría y son los que permitirán interpretar correctamente los datos derivados de dicho estudio.

\section{Adquisición de datos microtermométricos}

Los datos más comunes que se obtienen a partir de la microtermometría de inclusiones fluidas son (1) la temperatura del eutéctico del sistema químico, que informa sobre la complejidad química de los solutos, (2) la temperatura de fusión de la hidrohalita, que permite determinar la cantidad de $\mathrm{CaCl}_{2}$ relativa a la de $\mathrm{NaCl}$ en el sistema, (3) la temperatura de fusión del hielo, que permite calcular la salinidad total del fluido en inclusiones sin cristales hijos, (4) la temperatura de fusión de clatratos (hidratos de $\mathrm{CO}_{2}$, $\mathrm{CH}_{4}$, etc.), que permite identificar la presencia de algunos gases en las inclusiones, (5) la temperatura de solubilización de cristales hijos, que permite calcular la salinidad para determinados componentes en inclusiones con cristales hijos y (6) la temperatura de homogeneización. Acerca de esta última y de la salinidad se abunda a continuación. 
3.1. Temperatura de homogeneización y temperatura de atrapamiento

Uno de los condicionantes básicos que es preciso asumir antes del estudio microtermométrico de inclusiones fluidas es que éstas permanecieron como sistema cerrado desde su atrapamiento hasta su análisis en laboratorio, por lo que su masa debe ser constante. Dado que, para poder emprender dicho estudio, es también necesario que el volumen de cada inclusión haya permanecido constante, la densidad de cada inclusión será igualmente contante. La temperatura a la que se produce la homogeneización de una inclusión fluida (paso de fases líquida y vapor a una sola fase líquida, vapor, o supercrítica) se denomina temperatura de homogeneización. Ésta debe considerarse como la temperatura mínima a la que se atrapó una inclusión fluida, siempre y cuando el atrapamiento se haya producido a partir de un fluido homogéneo en cuanto a la composición de sus fases. La temperatura de atrapamiento será siempre igual o mayor a la de homogeneización. Esta última, junto con todas las posibles temperaturas de atrapamiento para la densidad de una inclusión fluida concreta, determinan una línea dentro del diagrama T-P de las fases correspondientes a los componentes de la inclusión fluida (por ejemplo, el del agua), que se denomina isocora. Ésta determina el rango de variación posible de presión y temperatura de atrapamiento para una densidad concreta a partir de la temperatura de homogeneización. La densidad (al igual que la salinidad), a su vez, es determinada mediante ecuaciones de estado correspondientes a las especies químicas identificables en las inclusiones fluidas, utilizando los datos microtermométricos en determinaciones de baja temperatura (esencialmente, la temperatura de fusión del hielo) y el grado de relleno o la temperatura de homogenización. Mediante la determinación de isocoras y la aplicación de geotermómetros o geobarómetros independientes pueden obtenerse, respectivamente, la presión a partir de la temperatura y viceversa. Ello siempre y cuando se pueda establecer que las temperaturas o presiones obtenidas mediante otros geotermómetros o geobarómetros son representativas de las condiciones físicas durante las que se produjo el atrapamiento de las inclusiones fluidas.

Es importante determinar las condiciones de atrapamiento de un conjunto de inclusiones fluidas y, por ende, de las asociaciones minerales que las contienen, especialmente para fines metalogenéticos. Sin embargo, a efectos de la exploración minera, tal determinación no es imprescindible, pues la obtención de un conjunto de datos espacial y temporalmente representativos (e internamente consistentes) es prioritaria y la obtención de temperaturas de homogeneización es suficiente. Ahora bien, ello no es aplicable en el caso de yacimientos que registren telescopaje (es decir, diversos eventos formados en condiciones físicas de orden decreciente) o yacimientos formados en rangos de profundidad muy amplios. Ello es debido al hecho que la geometría de las isocoras en el diagrama de fases de presión y temperatura del agua varía según su valor de densidad, de forma que pequeñas diferencias entre los valores de presión de atrapamiento y presión de homogeneización pueden corresponderse con grandes diferencias entre los valores de temperatura de atrapamiento y temperatura de homogeneización. Igualmente, pequeñas diferencias entre los valores de temperatura de atrapamiento y temperatura de homogeneización pueden corresponderse con grandes diferencias entre los valores de presión de atrapamiento y presión de homogeneización. Así, cabe remarcar que las confusiones en la adscripción de valores correspondientes a sistemas físico-químico-geológicos diferentes puede conllevar grandes errores en la estimación de las variables físicas en el momento del atrapamiento de las inclusiones fluidas y la no determinación de éstas puede enmascarar tales confusiones. Por ello, en situaciones en que los criterios geológicos básicos indiquen la posible existencia de telescopaje o cuerpos formados en un rango de profundidad muy amplio, es preferible determinar las condiciones de atrapamiento para cada evento $\mathrm{y} / \mathrm{o}$ en los diferentes rangos de profundidad de formación significativos.

Para el caso de sistemas con coexistencia de salmueras acuosas e hidrocarburos existe un protocolo analítico específico, detallado por González-Partida et al. (2008a). El caso de asociaciones de inclusiones fluidas que registran la existencia de ebullición durante su atrapamiento es particularmente interesante en microtermometría, pues permite obtener directamente la temperatura de atrapamiento mediante microtermometría de las inclusiones con el mayor grado de relleno (mayor proporción de líquido).

3.2. Importancia de la identificación de asociaciones de inclusiones fluidas de alta temperatura

Los sistemas hidrotermales naturales de cualquier tipo son sumamente dinámicos en el espacio y en el tiempo. Por ello, dentro de una misma asociación mineral, e inclusive a escala de cristal, las inclusiones fluidas pueden registrar grandes variaciones en temperatura de homogeneización y salinidad (Figura 2). A la hora de abordar el estudio microtermométrico sistemático de las diversas asociaciones de inclusiones fluidas de un cuerpo geológico (según una distribución espacio-temporal preestablecida), es preciso cuestionarse acerca de cuáles de las asociaciones de inclusiones fluidas presentes en las muestras problema son las más indicadas para dicho estudio. Éste puede tener (1) un propósito académico, en que prima la adquisición de datos exhaustivos para caracterizar debidamente el máximo de eventos geológicos asociados a la formación de un depósito mineral, o bien (2) un propósito exploratorio, en que prima la adquisición de datos relevantes y útiles para guiar la posible explotación futura de un yacimiento mineral. Ambos propósitos requieren de una cierta economía de recursos (incluido el tiempo), pero la exploración minera requiere de tiempos de análisis menores que un estudio puramente académico. En ambos casos la selección de aso- 


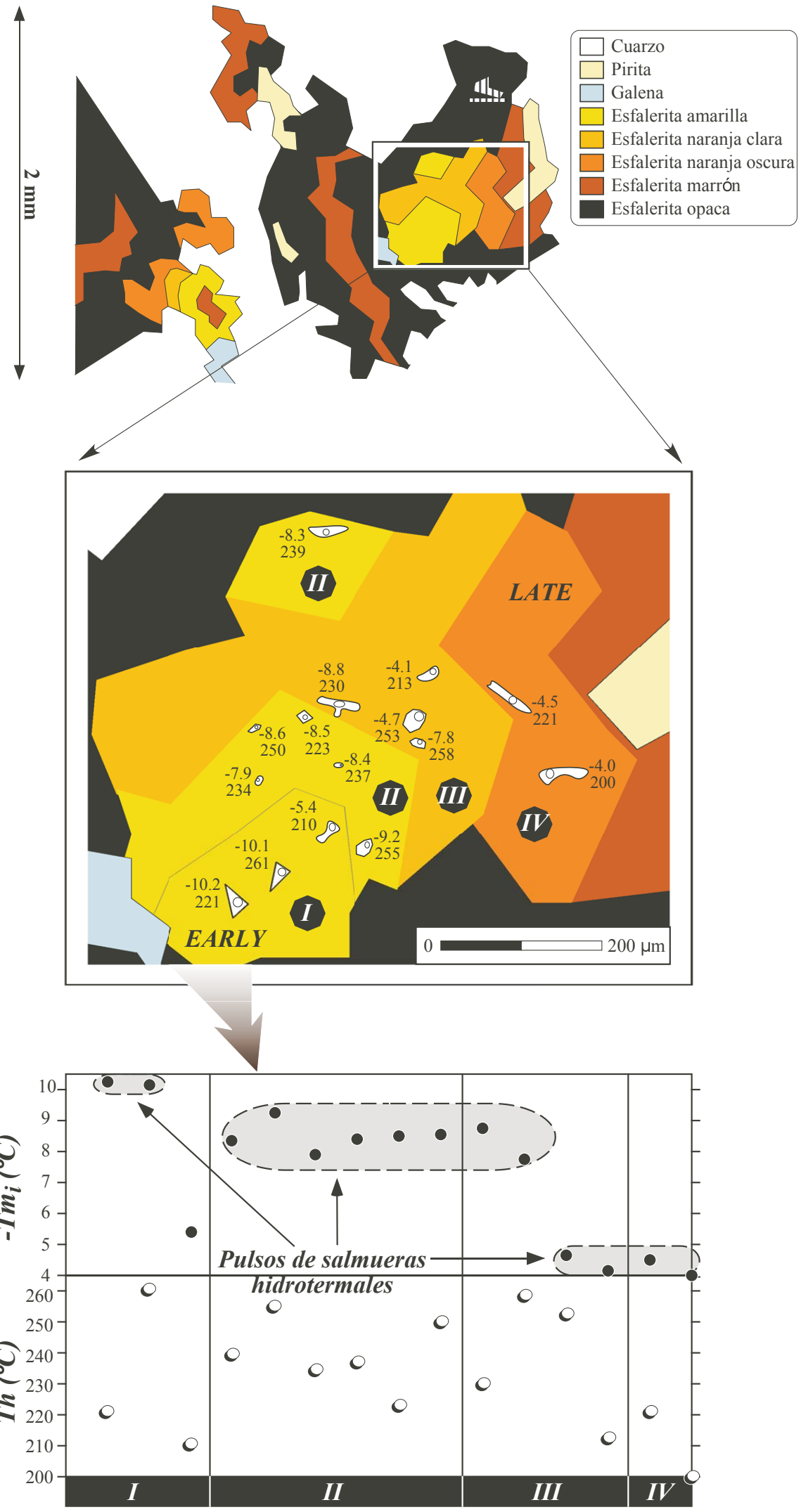

Figura 2. Ejemplo de la variación en temperaturas de homogeneización y de fusión de hielo a microescala en un cristal zonado de esfalerita de la etapa I en el yacimiento epitermal La Guitarra (distrito de Temascaltepec, Estado de México). Adaptado de Camprubí et al. (2001b). Clave: Tmi = temperatura de fusión del hielo, $\mathrm{Th}=$ Temperatura de homogeneización. 
ciaciones de inclusiones fluidas a analizar es crucial, pues siempre es preciso escoger asociaciones representativas de los diversos eventos registrados en el cuerpo geológico sujeto a análisis, pero es un aspecto especialmente crítico en el caso de la exploración minera. Dicho de otra forma, no es metodológicamente correcto realizar un análisis indiscriminado, sino que un propósito exploratorio requiere un mayor sentido selectivo, pues de lo representativo hay que obtener una cantidad de datos significativos aún más restringidos que para un estudio académico. Por ello, es recomendable seleccionar preferentemente para su análisis microtermométrico asociaciones de inclusiones fluidas de alta temperatura, pues éstas son representativas de microeventos térmicamente climáticos en cualquier pulso hidrotermal. La representatividad de las inclusiones fluidas de más alta temperatura es mayor que las de baja temperatura porque estas últimas no son tan distintivas de los pulsos hidrotermales como las primeras. Es decir, la temperatura más característica de un evento hidrotermal en un lugar concreto será siempre la máxima (ver pie de la Figura 6 en Albinson et al., 2001).

La selección preferencial de asociaciones de inclusiones fluidas de alta temperatura para propósitos exploratorios, sin embargo, no debe realizarse en detrimento de asociaciones de más baja temperatura. Al contrario, la selección debe incluir asociaciones de inclusiones representativas de las presentes en las muestras problema, pero siempre deberá incluir asociaciones de la más alta temperatura posible. Lógicamente, éstas se distinguen por presentar grados de relleno relativamente bajos (relaciones líquido/vapor bajas) de forma consistente a través de una asociación de inclusiones fluidas determinada petrográficamente.

\subsection{Significado y utilidad de la salinidad}

La salinidad es, junto con las temperaturas de homogeneización/atrapamiento, la variable fisicoquímica más característica que se puede obtener por medio de la microtermometría de inclusiones fluidas. La salinidad representa el conjunto de solutos que se encontraban en solución dentro de un solvente acuoso en el momento del atrapamiento de una inclusión fluida. Ello implica que en las inclusiones en que se haya producido la precipitación de cristales hijos la salinidad no es la del líquido restante, sino la del líquido toda vez que se ha producido la disolución total de los cristales hijos. Es de suma importancia poder determinar, mediante criterios petrográficos (y en parte microtermométricos), si los cristales presentes en una inclusión fluida precipitaron en su interior (cristales hijos) o bien fueron atrapados accidentalmente a partir de un fluido en el que se encontraban en suspensión (cristales atrapados): los primeros entran dentro del cómputo de la salinidad de los fluidos mineralizantes, los segundos no.

La salinidad y la composición química de los solutos permiten evaluar la capacidad de transporte de los fluidos mineralizantes y mediante qué tipo de complejos químicos solubles pudo producirse el transporte de los diversos metales. Los complejos químicos pueden ser muy diversos (tiosulfuros, cloruros, amonio, etc.) y, al igual que los minerales, su estabilidad depende de las condiciones fisicoquímicas del medio (temperatura, presión, $f \mathrm{O}_{2}, f \mathrm{~S}_{2}$, etc.) y, en algunos casos, ayudan a acotar el origen de los fluidos. La mineralogía de los cristales hijos es identificable por lo general mediante la morfología externa de éstos y por su comportamiento óptico, lo cual permite determinar inicialmente la composición de los solutos mayoritarios en los fluidos mineralizantes. En cambio, en inclusiones fluidas que carecen de cristales hijos ello no es posible. Sin embargo, la cuantificación de los solutos relativa a la cantidad de solvente, o salinidad, debe realizarse necesariamente mediante análisis microtermométrico. Generalmente, la salinidad se expresa como tanto por ciento en peso equivalente de $\mathrm{NaCl}$ (wt.\% $\mathrm{NaCl}$ equiv.), por ser éste el soluto mayoritario en la mayoría de sistemas hidrotermales naturales. Asimismo, la temperatura eutéctica nos da información acerca del tipo de sales presentes en la inclusión (Crawford, 1981). Por otro lado, la determinación de la presencia de diferentes cationes y aniones y sus relaciones molares se realiza mediante muy diversas técnicas geoquímicas avanzadas (ver relación de algunas de ellas en Wilkinson, 2001, página 237). Los cationes mayoritarios en los fluidos acuosos naturales son $\mathrm{Na}, \mathrm{K}$ y $\mathrm{Ca}$, seguidos por $\mathrm{Fe}$ y $\mathrm{Mg}$, mientras que el anión más frecuente es $\mathrm{Cl}^{-}$, con cantidades menores de $\mathrm{SO}_{4}{ }^{2-}, \mathrm{HCO}_{3}^{-} \mathrm{y}$ $\mathrm{NO}_{3}^{-}$(Wilkinson, 2001). De todos ellos, el de mayor importancia metalogenética es el $\mathrm{Cl}^{-}$, pues es el principal ión complejante para el transporte de metales, especialmente metales básicos y Ag.

Mediante el análisis microtermométrico sistemático de diversas etapas de mineralización en diversas posiciones dentro de los depósitos minerales es posible reconstruir la evolución fisicoquímica de los fluidos mineralizantes a través del tiempo (Figura 1); determinar diversos mecanismos de precipitación mineral para asociaciones minerales diversas (Figura 3, 4 y 5, ver sección 6); determinar patrones de interacción entre fluidos de diferentes orígenes o con diferentes contenidos en solutos (Figura 3); determinar la presencia de diversos pulsos o micro-pulsos hidrotermales en una misma asociación mineral (Figura 2); y determinar la existencia de falsas salmueras debidas a ebullición (Figura 3).

Además, es esencial determinar la salinidad de los fluidos para realizar adecuadamente estimaciones de la posición de paleoniveles freáticos (o de paleosuperficies), como se verá más adelante.

En yacimientos epitermales, la determinación de la salinidad es uno de los factores esenciales a tener en cuenta para la determinación de las diversas subtipologías de éstos (yacimientos de sulfuración alta, intermedia y baja). Dentro de un mismo depósito pueden encontrarse más de una de estas subtipologías (Camprubí y Albinson, 2006, 2007). Por ello, teniendo en cuenta que las diferentes sub- 


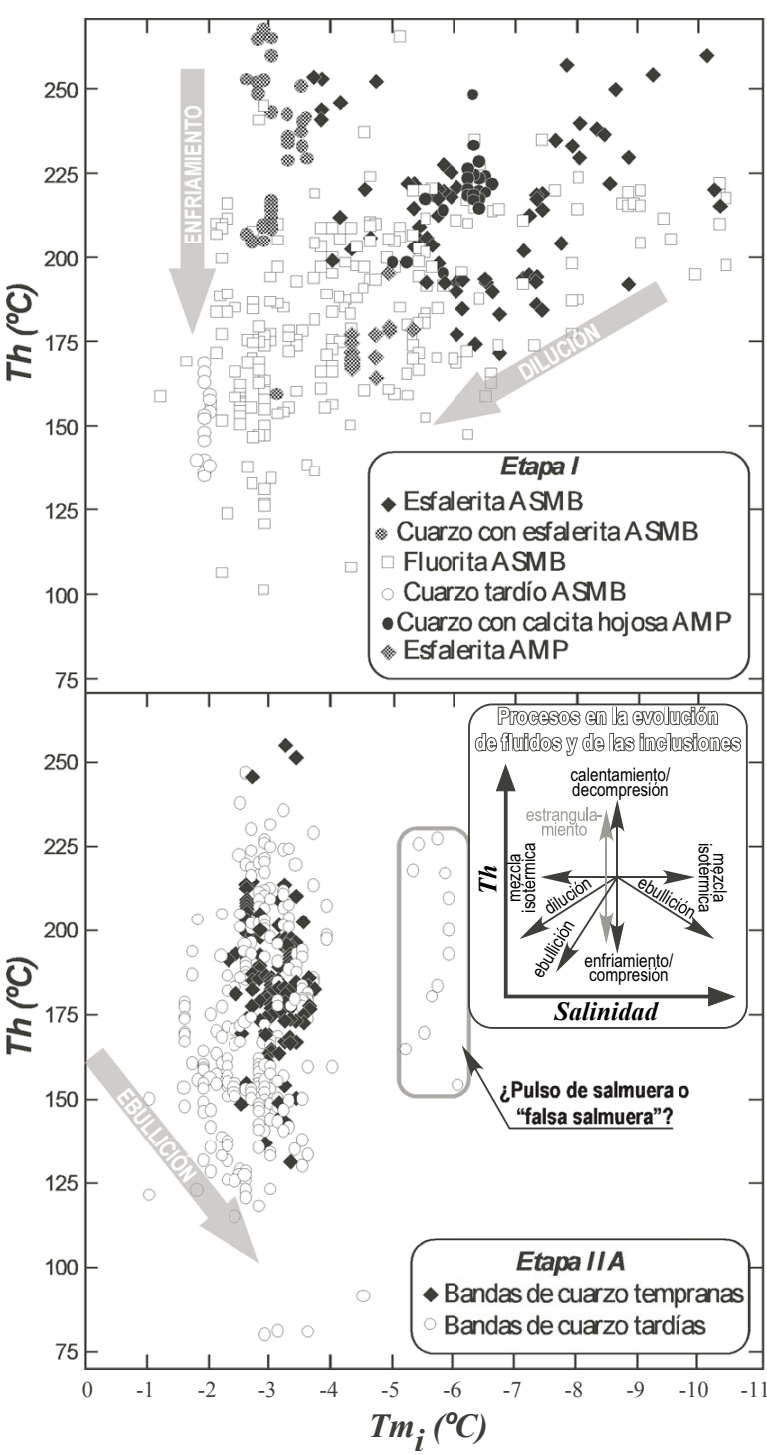

Figura 3. Correlación entre la temperatura de fusión de hielo $\left(\mathrm{Tm}_{\mathrm{i}}\right)$ y la temperatura de homogeneización (Th) de inclusiones fluidas individuales en dos etapas de mineralización del yacimiento epitermal de La Guitarra (distrito de Temascaltepec, Estado de México), cuya distribución sugiere la existencia de diversos procesos de mineralización. Modificado de Camprubí et al. (2001b). El recuadro en el interior del diagrama de la etapa IIA se modificó de Wilkinson (2001). Clave: ASMB = asociaciones de sulfuros de metales básicos, AMP = asociaciones con minerales de plata.

tipologías y diferentes estilos de mineralización presentan típicamente diferentes asociaciones metálicas, la simple determinación de la salinidad en un conjunto representativo de muestras de la misma etapa de mineralización constituye un criterio de exploración importante (Figura 6).

En fluidos de baja salinidad que presentan contenidos de $\mathrm{CO}_{2}$ "moderados", en el orden de varias unidades porcentuales en peso de gas disuelto, la contribución del $\mathrm{CO}_{2}$ en la temperatura de fusión del hielo puede ser dominante (Wilkinson, 2001), aunque no lleguen a formarse clatratos de ese gas. De esa forma, en el caso de producirse ebulli- ción o efervescencia en una salmuera acuosa, la migración preferencial del $\mathrm{CO}_{2}$ hacia la fase gaseosa puede provocar que las inclusiones fluidas que hayan atrapado parte del líquido residual registren una temperatura de fusión del hielo mayor que las inclusiones fluidas que atraparon porciones del fluido anterior a la ebullición o efervescencia (Wilkinson, 2001). Así, en una secuencia de asociaciones de inclusiones fluidas que registren parte del fluido precursor y del fluido residual pudiera registrarse una aparente disminución de salinidad cuando en realidad las características químicas reales de los dos fluidos serían (1) fluido precursor con baja salinidad y contenido en $\mathrm{CO}_{2}$ moderado, y (2) fluido residual con salinidad moderada o media y contenido en $\mathrm{CO}_{2}$ muy bajo. Para evitar estimaciones erróneas de la salinidad a partir de la temperatura de fusión del hielo, es recomendable (si es ello posible) realizar determinaciones mediante técnicas no microtermométricas del contenido en $\mathrm{CO}_{2}$, como la microespectroscopía Raman.

En depósitos metalíferos asociados a pórfidos, la caracterización sistemática de las salinidades en asociaciones de inclusiones fluidas con cristales hijos es crítica para la determinación de (1) la evolución físico-química de dichos sistemas, (2) la identificación de salmueras anteriores a la "primera ebullición" o "ebullición magmática", (3) la presencia de sólidos atrapados, frecuentemente con la misma mineralogía que los posibles cristales hijos, así como (4) de la existencia de procesos de modificación post-atrapamiento (e. g., Harris et al., 2005; Becker et al., 2008).

\section{Uso y utilidad de las inclusiones fluidas en exploración}

4.1. Determinación de tipologías o subtipologías de depósitos minerales

El uso más inmediato de las inclusiones fluidas para fines exploratorios reside en el establecimiento empírico de la relación entre ciertas propiedades microtermométricas de las inclusiones fluidas y características clave de las mineralizaciones económicas (Wilkinson, 2001). En primer lugar, los rangos de temperatura o de salinidad genéricos se pueden considerar (1) como orientativos en la determinación de una tipología o subtipología de yacimientos minerales (Figuras 6 y 7) o (2) como reveladores de un ambiente hidrotermal permisivo para mineralizaciones económicas. En la determinación tipológica de los depósitos minerales, la petrografía y microtermometría de inclusiones fluidas constituyen un elemento de juicio cualitativo importante. Como ejemplo de un estudio reciente, es notable el caso del yacimiento de $\mathrm{Zn}-\mathrm{Cu}-\mathrm{Pb}(-\mathrm{Ag})$ Francisco I. Madero (Zacatecas, México central). La determinación sistemática de la mineralogía de las diversas facies constitutivas de dicho yacimiento (Canet et al., 2009), junto con la microtermometría de inclusiones fluidas (datos inéditos), permitió determinar que dicho yacimiento se formó a partir 


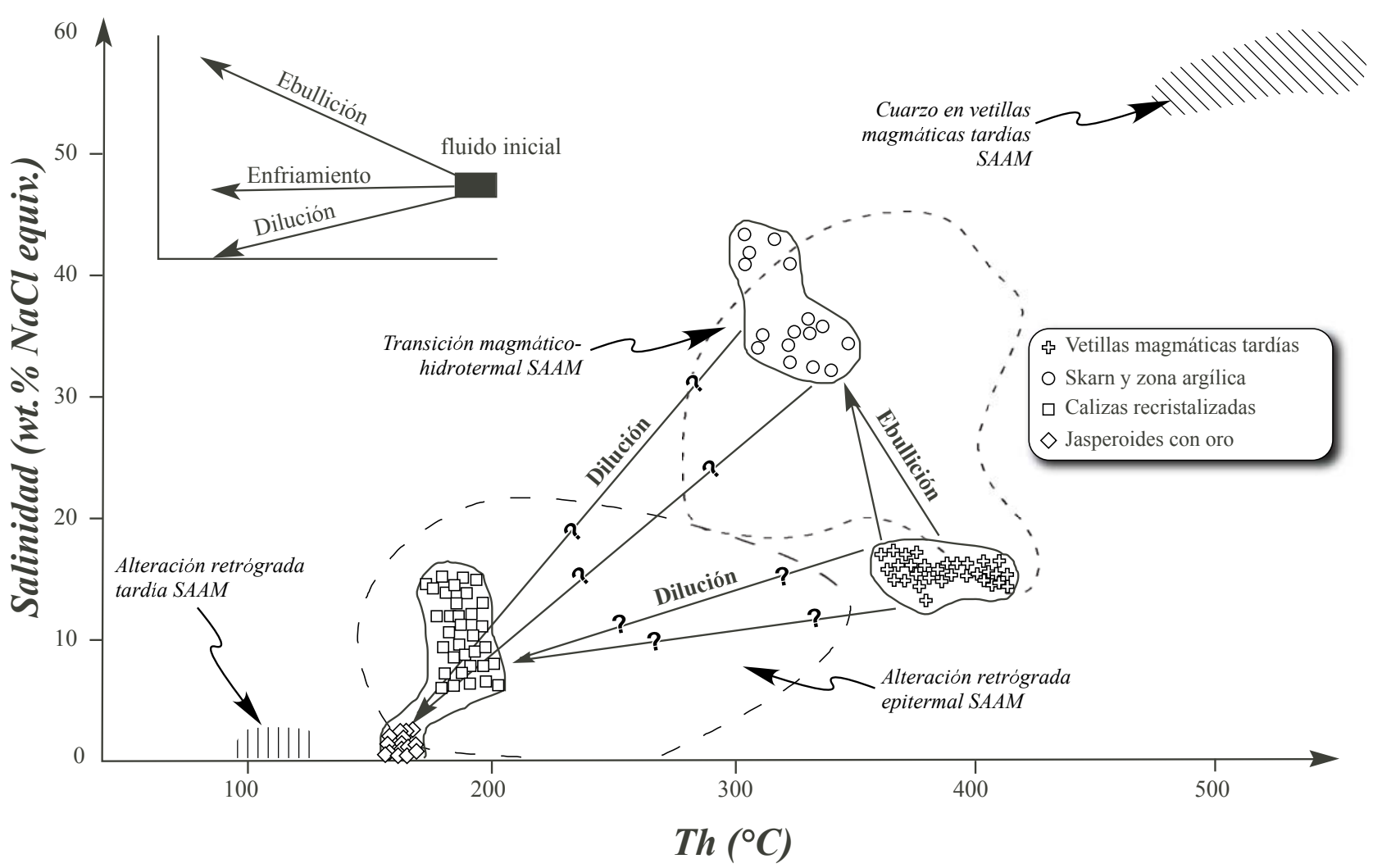

Figura 4. Diagrama de correlación entre la temperatura de homogeneización de inclusiones fluidas con su salinidad (González-Partida et al., 2004), obtenidas en el depósito de tipo skarn de La Joya (distrito de Mezcala, Guerrero), mostrando las posibles tendencias de evolución de los fluidos magmáticos iniciales (cuadro superior izquierdo), a partir de mecanismos de (1) enfriamiento, en que el fluido permanece iso-salino a medida que la temperatura baja, proceso que no se ha reconocido en La Joya; (2) dilución por parte de aguas meteóricas, en que los fluidos iniciales se enfrían a la par que disminuye su salinidad; y (3) ebullición, en que aumenta drásticamente la salinidad de las salmueras debido al escape de vapor, y su temperatura disminuye debido a la descompresión. Las áreas delineadas corresponden a los datos obtenidos en diversos skarns ricos en Au asociados a adakitas en el distrito de Mezcala. Clave: SAAM = Skarns auríferos en adakitas de Mezcala, $\mathrm{Th}=$ Temperatura de homogeneización.

de reemplazamientos a alta temperatura (temperaturas de homogeneización hasta $525^{\circ} \mathrm{C}$ en la parte basal del depósito). Dicho esquema genético, análogo a ciertos tipos de skarns, es muy distinto a interpretaciones previas basadas en modelos genéticos tipo SEDEX o volcanogénicos, ante los cuales las estrategias de exploración locales y regionales son necesariamente distintas.

En el caso que las variables microtermométricas de las inclusiones fluidas puedan ser reveladoras de mineralizaciones económicas, los criterios de guía en base a rangos de temperatura o de salinidad varían en función del tipo de yacimiento mineral y, en algunos casos, varían entre yacimientos distintos de la misma tipología. Por ello, es necesario que el prospector posea un conocimiento suficiente acerca del tipo de yacimiento que se encuentra bajo exploración, no sólo acerca de la zonación mineral o metálica esperable para cada tipología, sino también acerca de los tipos de zonación térmica y las características petrográficas y fisicoquímicas de las inclusiones fluidas que pueden presentar. Asimismo, es preciso poseer conocimientos suficientes para poder interpretar el significado de la geometría de las isotermas determinadas a partir del estudio microtermométrico de inclusiones fluidas, o a través de otros geotermómetros (paragenéticos, composicionales, isotópicos, etc.). Wilkinson (2001) identificó las características petrográficas y fisicoquímicas genéricas de las inclusiones fluidas y, por extensión, de los fluidos mineralizantes en algunas de las tipologías de yacimientos minerales económicamente más importantes (Figura 5 y Tabla 2 en Wilkinson, 2001). Igualmente, dicho autor ilustra los principales procesos de mineralización en dichas tipologías y las variaciones en temperatura y composición de las inclusiones fluidas y sus fases constitutivas. Entre otras particularidades, dicho autor hace hincapié en (1) la alta densidad y baja temperatura de las salmueras mineralizantes en depósitos tipo MVT, (2) la notoria mayor temperatura de los fluidos en depósitos de $\mathrm{Pb}-\mathrm{Zn}-\mathrm{Ag}$-Ba de "Tipo Irlandés" que en MVT, (3) la mayor temperatura y salinidad de fluidos en stockworks que en la parte superior de los depósitos de sulfuros masivos vulcanogénicos tipo Kuroko, (4) el amplio rango de variación de temperatura y salinidad, así como en la mineralogía de cristales hijos en 

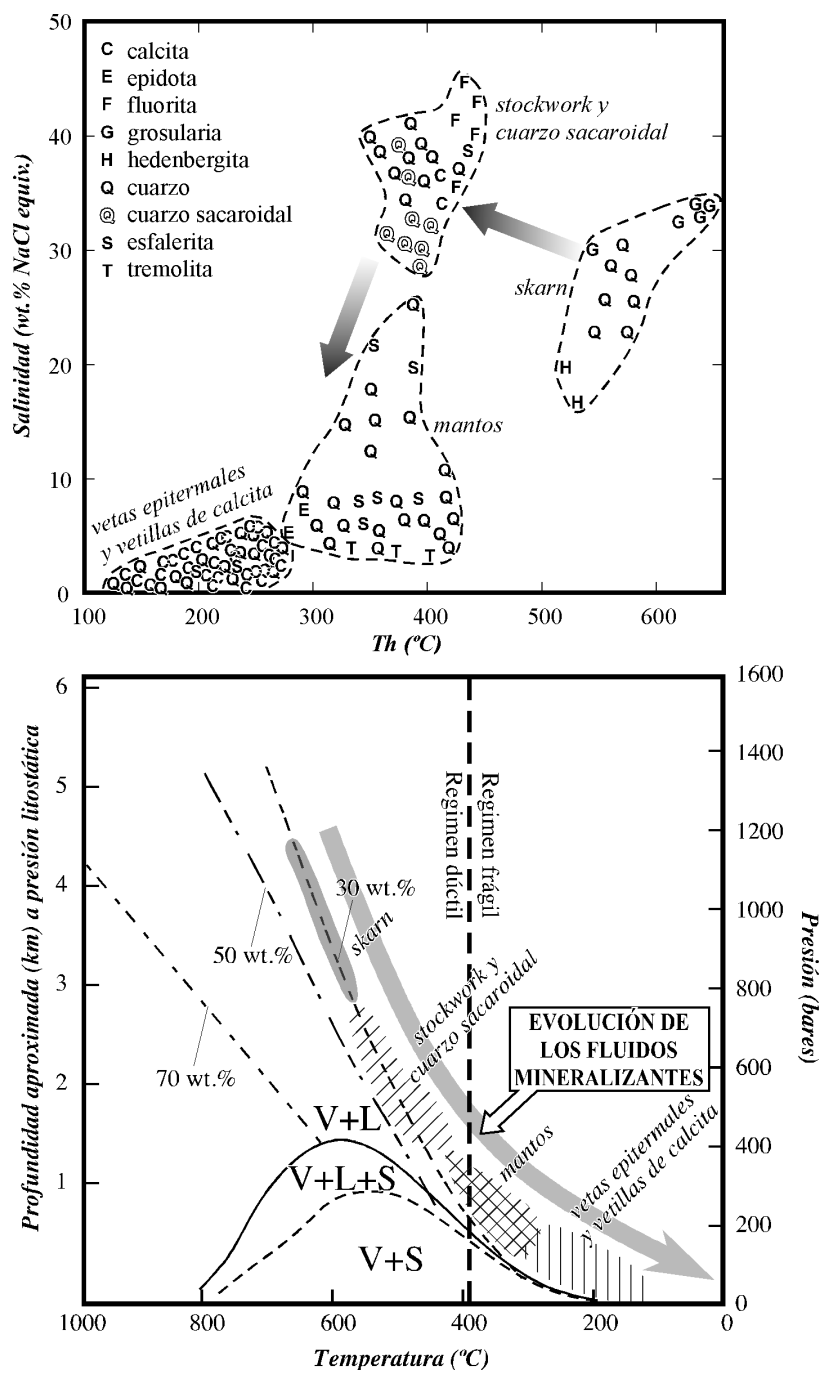

Figura 5. Arriba: Diagrama de correlación entre valores promedio de temperatura de homogeneización y salinidad por muestra, agrupados de acuerdo a las principales asociaciones minerales. Abajo: Diagrama de correlación entre temperatura y profundidad de fluidos mineralizantes en los depósitos de tipo skarn del distrito de San Martín (Zacatecas), mostrando las relaciones de fases en el sistema agua- $\mathrm{NaCl}$ a presión litostática y curvas de isosalinidad clave (30, 50 y $70 \mathrm{wt} . \% \mathrm{NaCl}$ equiv.), adaptado de Fournier (1999). Clave: $\mathrm{L}=$ líquido, $\mathrm{S}=$ sólido (halita), $\mathrm{V}$ = vapor. Adaptado de González-Partida y Camprubí (2006). Clave: $\mathrm{Th}=$ Temperatura de homogeneización.

depósitos de Sn-W-Cu asociados a granitoides y en depósitos metalíferos (Cu-Au-Mo) en pórfidos, (5) la práctica correspondencia entre temperatura de homogeneización y de atrapamiento en depósitos epitermales y (6) la ubicuidad del $\mathrm{CO}_{2}$ en depósitos mesotermales.

4.2. Identificación de temperaturas favorables a la presencia de mineralizaciones

Ante todo, es preciso remarcar que no siempre es posible asociar un rango de temperaturas de homogeneización (o atrapamiento) directamente a la presencia o ausencia de mineralizaciones económicas, ni teórica ni empíricamente. Sin embargo, en ocasiones es posible establecer una "asociación preferencial" entre ambas, aunque se trata de relaciones empíricas que normalmente requieren de la distinción de los datos procedentes de asociaciones de inclusiones fluidas de alta temperatura. Estas relaciones empíricas suelen poderse usar como criterio de exploración a escala de yacimiento o de distrito aunque, en algunos casos, su uso puede generalizarse a yacimientos tipológicamente afines. Adicionalmente, dado que se trata de relaciones establecidas de forma empírica, lo más prudente al respecto es usar datos mineralógicos de apoyo, como la presencia de asociaciones minerales características (Figura 5 y 8). Este tipo de relaciones no puede establecerse de forma inmediata, pues por lo común precisa de estudios petrográficos y microtermométricos completos.

\subsection{Identificación de canales de ascenso de fluidos}

La zonación térmica de los yacimientos, determinada según un patrón de isotermas de temperaturas significativas, permite la identificación de canales de ascenso de fluidos y de zonas de dispersión hidrotermal (Figura 9). Dichos canales o zonas de alimentación preferencial se identifican por la presencia de domos térmicos (según el trazado de las isotermas) a cuya elevación en otras áreas se encuentran isotermas de menor temperatura. Por lo común, se considera que cuanto más pronunciado es el domo térmico, más probable es la existencia de un canal de ascenso hidrotermal preferencial. Para la determinación lo más certera posible de patrones de este tipo es imprescindible haber caracterizado con detalle previamente la zonación del depósito, al menos en términos de secuencia temporal. Ello, a efectos de consignar debidamente la información microtermométrica a cada etapa o subetapa de formación del depósito. En ocasiones, los patrones de distribución de isotermas entre diferentes etapas no son en absoluto similares. Ello no constituye necesariamente un elemento de sospecha acerca de la validez de los datos o del tratamiento de éstos. Sin embargo, en los casos en que los patrones de distribución de las isotermas son similares de una etapa a otra, puede considerarse (especialmente en los casos en que patrones similares son debidos a datos de muestras distintas) que (1) los datos de una etapa "validan" comparativamente los datos de otra etapa y que, por tanto, (2) la presencia de canales de ascenso hidrotermal u otros patrones térmicos es consistente en el tiempo, por lo que pueden derivarse interpretaciones de índole estructural.

La determinación de la presencia de canales preferenciales de ascenso hidrotermal radica en el hecho que, cuando éstos pueden relacionarse espacial y temporalmente con la presencia de zonas de mineralización económica o ciertos tipos de asociaciones minerales (e.g. como evidencias de ebullición, de incursión de fluidos hipogénicos ácidos, etc.), constituyen una poderosa guía de exploración a nivel 


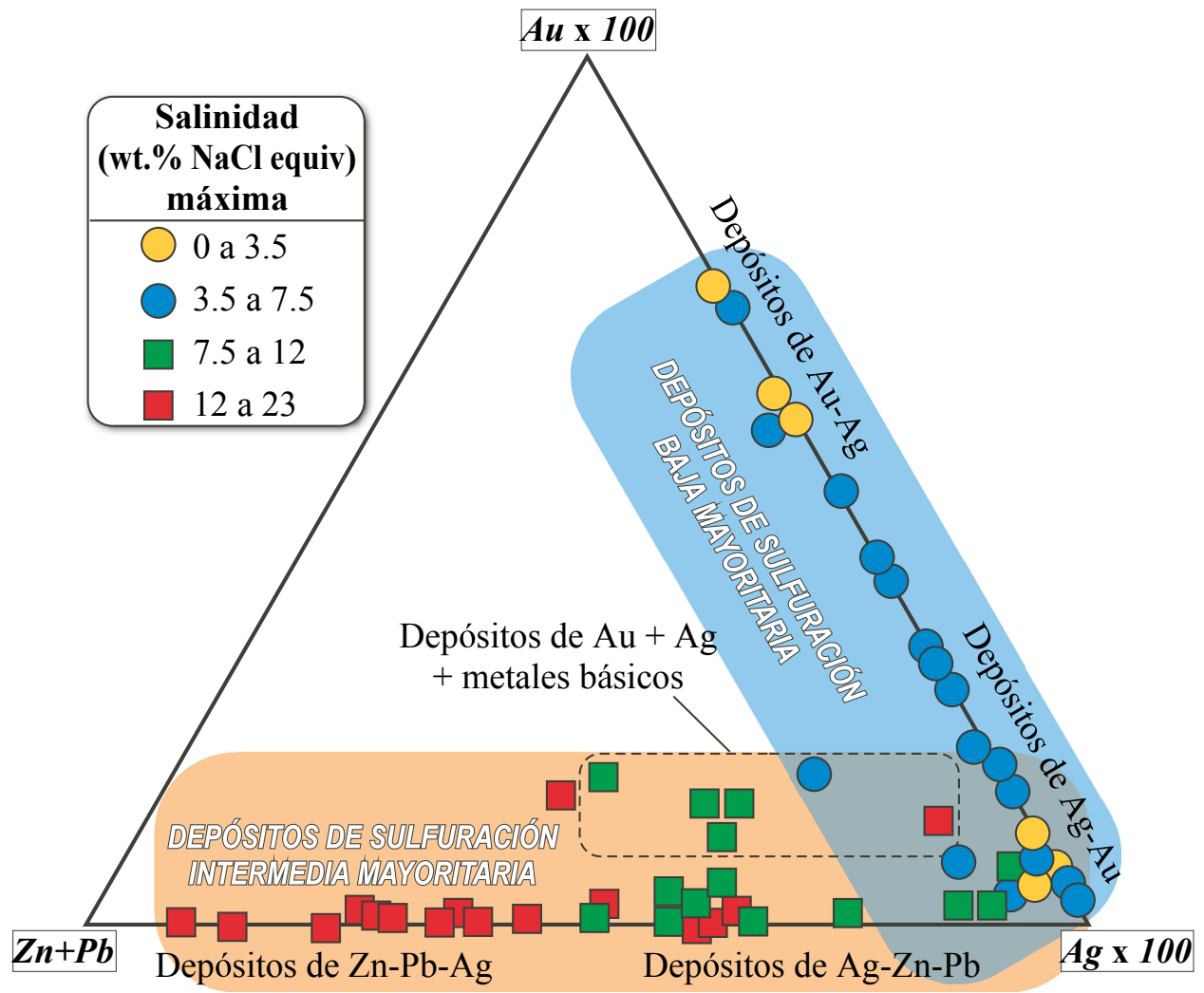

Figura 6. Diagrama que muestra las relaciones en contenidos metálicos de numerosos yacimientos epitermales de México, con indicación de las salinidades máximas de los fluidos mineralizantes asociados a éstos. Adaptado de Albinson et al. (2001).

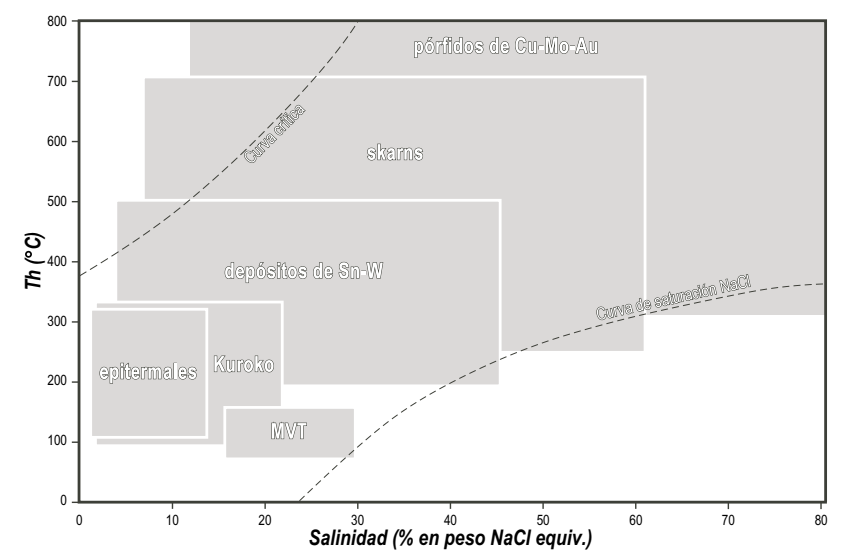

Figura 7. Rangos genéricos de temperatura y salinidad de tipologías escogidas de depósitos minerales. Adaptado de Wilkinson (2001). Clave: MVT = depósitos tipo Mississippi Valley, Th = Temperatura de homogeneización.

de yacimiento. En algunos casos, los canales de ascenso se encuentran confinados por la presencia de diques (Figura 9B) u otras litologías relativamente favorables o desfavorables al desarrollo de fracturamiento y/o de circulación de fluidos, de forma que la mera presencia de éstas puede constituir una guía de exploración. La presencia de canales de ascenso puede refrendarse mediante métodos de apoyo tales como los cocientes $\mathrm{Ag} / \mathrm{Pb}$ en la mineralización y las relaciones $\mathrm{Sb} /(\mathrm{Sb}+\mathrm{As})$ en tetraedrita-tennantita (Goodell y Petersen, 1974; Wu y Petersen, 1977; Camprubí et al., 2001a). Una vez identificados los canales de ascenso principales en un yacimiento se puede investigar la posible continuidad del yacimiento a mayor profundidad, pues ésta se encontrará probablemente centrada en dichos canales. Los yacimientos epitermales, por ejemplo, pueden presentar raíces polimetálicas aunque a mayor elevación sean ricos en metales preciosos e, inclusive, estas raíces pueden estar físicamente desconectadas de las porciones más someras del yacimiento (Camprubí y Albinson, 2007).

\subsection{Elementos de apoyo mineralógicos y estructurales}

A pesar de que los datos microtermométricos y su distribución espacial (para cada etapa de mineralización en depósitos polifásicos y multiepisódicos) constituyen una poderosa herramienta de exploración, existen numerosos criterios mineralógicos y estructurales capaces de aportar elementos de discusión adicionales.

Los elementos mineralógicos a relacionar para ser usados como criterios adicionales comprenden tanto la mineralogía de los halos de alteración hipogénica (Figura 


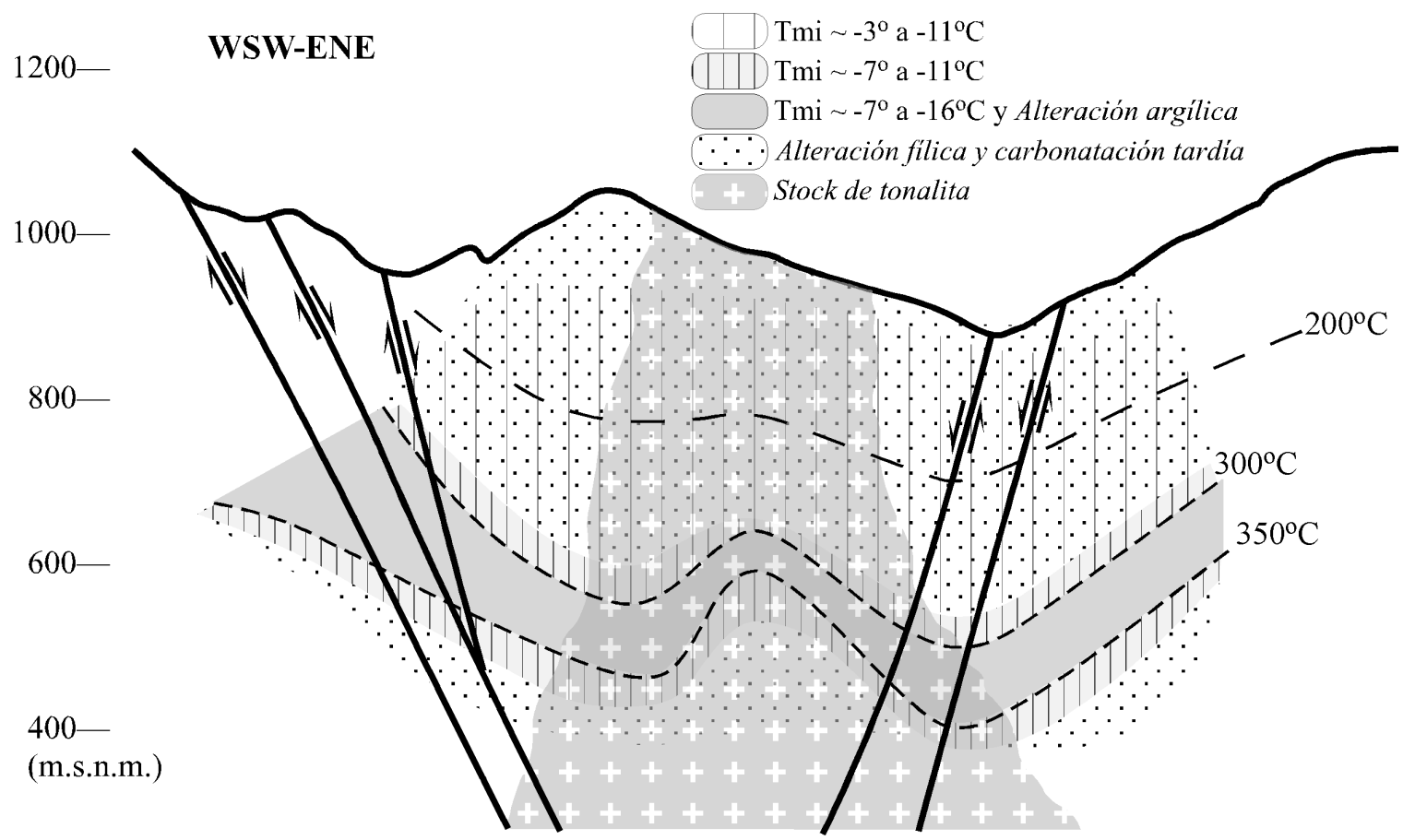

Figura 8. Sección geológica del pórfido cupro-aurífero del distrito de Tiámaro (Michoacán) mostrando la distribución de isotermas determinadas mediante temperaturas de homogeneización de inclusiones fluidas, los rangos de temperatura de fusión de hielo $\left(\mathrm{Tm}_{\mathrm{i}}\right)$, la distribución general de las asociaciones de alteración y la posición de un stock tonalítico. Adaptado de Garza-González et al. (2006). Clave: m.s.n.m. = metros sobre el nivel del mar. Clave: Tmi $=$ temperatura de fusión del hielo.

8) como la mineralogía o las asociaciones metálicas de los mismos cuerpos mineralizados (Figura 6). Tales elementos pueden agruparse en dos categorías: (1) la presencia de un mineral o asociación mineral diagnóstica de una temperatura o ambiente químico concreto (e. g. caolinita \pm alunita \pm dickita, asociación adularia + epidota), sin constituirse necesariamente en geotermómetros precisos y (2) el diámetro máximo y la morfología de los cristales y agregados cristalinos de un mineral común (e.g. clorita, hematites, epidota), pues su abundancia en el depósito permite relacionar sus características con una temperatura relativa. Por ejemplo, en algunos yacimientos epitermales de sulfuración intermedia y baja de México se ha observado la presencia de una estrecha relación paragenética entre adularia y epidota en asociaciones minerales tempranas de etapas portadoras de mineralizaciones ricas en metales básicos y plata, a temperaturas $\sim 300^{\circ} \mathrm{C}$. En algunos yacimientos, como en la Veta Zuloaga del distrito de San Martín de Bolaños (Jalisco), se ha identificado una zona de alteración argílica profunda bajo las zonas mineralizadas, a temperaturas $\sim 300^{\circ} \mathrm{C}$ (Albinson y Rubio, 2001; Camprubí y Albinson, 2007). Asimismo, el conjunto de tipologías de yacimientos en pórfidos metalíferos - skarns - epitermales se ha beneficiado de estudios mineralógicos en asociaciones de alteración hidrotermal en campos geotérmicos activos (e.g. Reyes, 1990, 1991).

Entre los elementos estructurales, cabe distinguir entre la estructura interna del yacimiento (e. g. la "estratigrafía" de vetas) y la disposición del mismo en el espacio (morfología, orientación e inclinación y cambios en éstos). La estructura interna del yacimiento es la que permite establecer inicialmente la secuencia de eventos que, a su vez, determina la ubicación temporal de los datos microtermométricos (Figura 1). En cambio, la disposición del yacimiento en el espacio determina no sólo la morfología o la posición de las zonas con mineralización metálica sino que puede llegar a determinar la misma presencia de éstas. En yacimientos determinados por vetas o conjuntos de vetas (e g. depósitos de tipo epitermal, algunas zonas de los skarns y de los depósitos vulcanogénicos de sulfuros masivos o vetas de $\mathrm{Sn}-\mathrm{W}$ ), las fracturas o fallas de bajo ángulo son poco favorables a albergar vetas con mineralización económica, contrariamente a las vetas de alto ángulo. Igualmente, los yacimientos epigenéticos que se desarrollan en forma de mantos (e. g. algunos depósitos epitermales, algunos skarns o depósitos tipo Mississippi Valley o MVT) precisan de litologías favorables para su emplazamiento, con lo que su control es más litológico que estructural. Así, es común que los depósitos MVT $\mathrm{y}$ asociados se formen a favor de carbonatos arrecifales o unidades evaporíticas (e. g., González-Sánchez et al., 2007). Por todo ello, algunos depósitos pueden presentar una combinación de efectos estructurales y litológicos sobre la presencia de mineralizaciones económicas que es preciso evaluar de forma individual. 

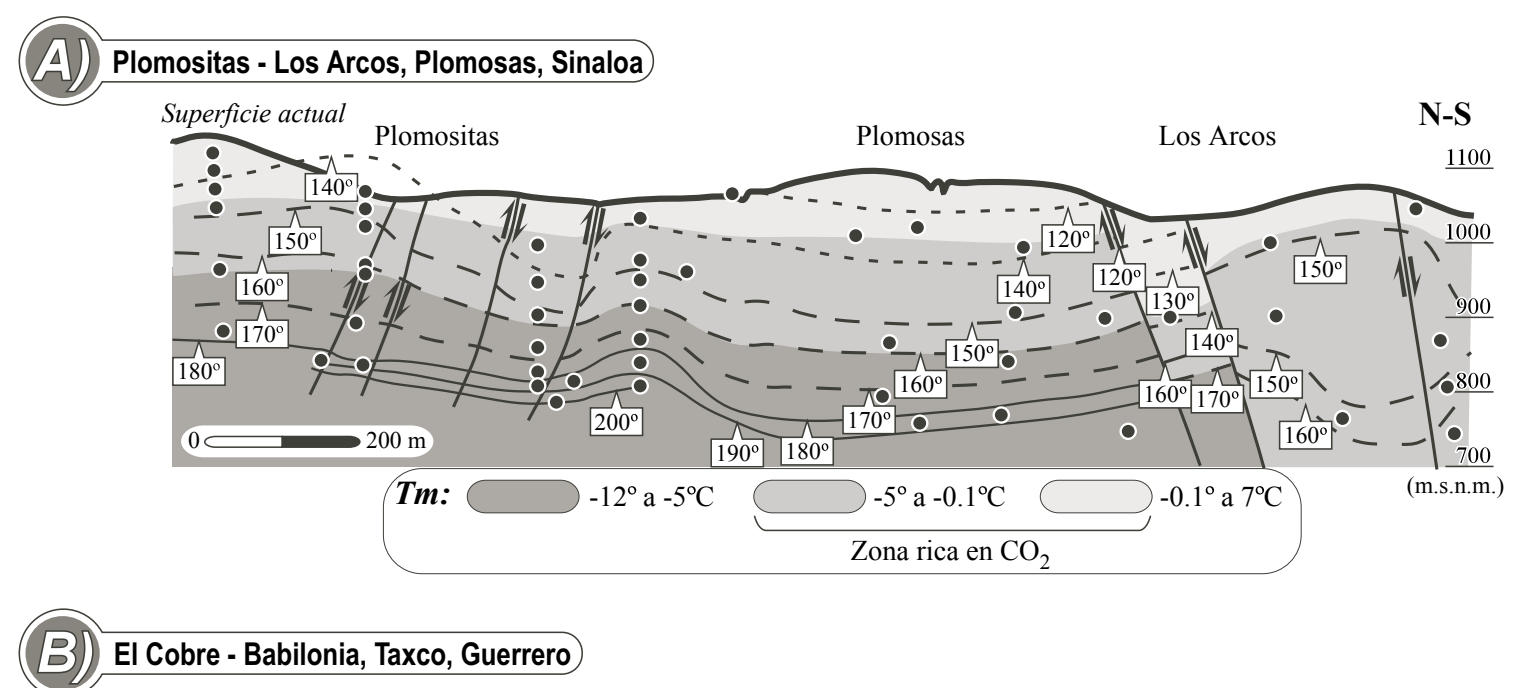

\section{El Cobre - Babilonia, Taxco, Guerrero}

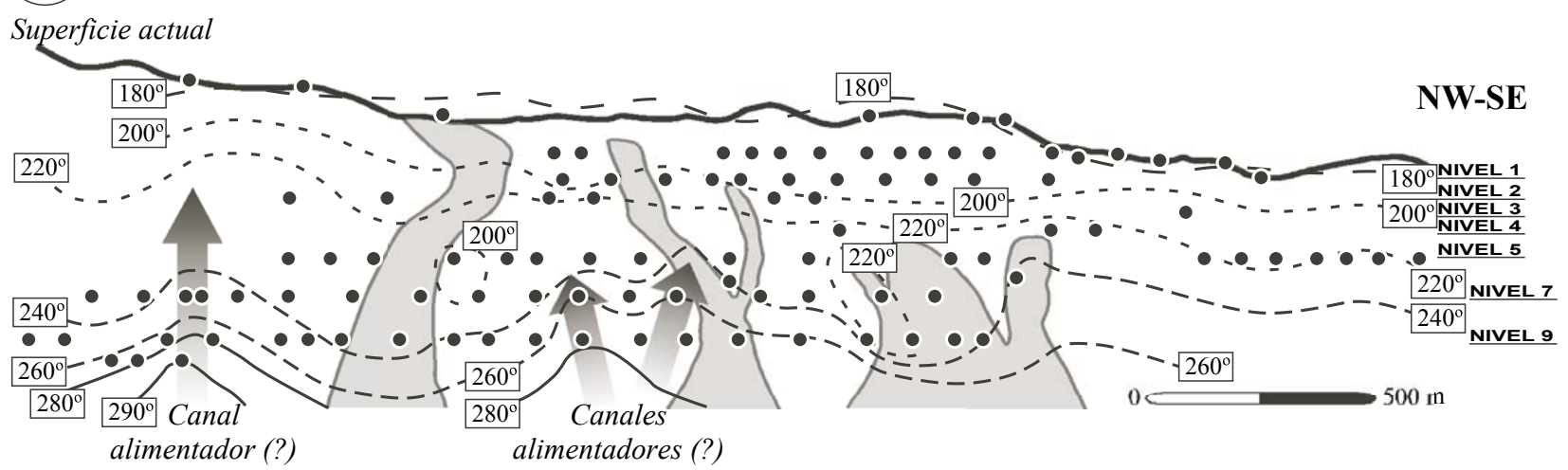

Figura 9. A: Sección longitudinal del cortejo de vetas epitermales Plomositas - Plomosas - Los Arcos (distrito de Plomosas, Sinaloa). Las altas temperaturas de fusión del hielo y la fusión de clatratos en la parte superior del depósito indican la presencia de abundante $\mathrm{CO}_{2}$. B: Sección longitudinal del cortejo de vetas epitermales El Cobre - Babilonia (distrito de Taxco, Guerrero). En color gris claro, se indica la presencia de diques anteriores a las vetas, a los que éstas intersectan. Ambas secciones presentan la localización de las estaciones de muestreo (puntos negros) y las isotermas construidas a partir de las temperaturas de homogeneización de inclusiones fluidas. Todas las temperaturas se reportan en grados centígrados. Adaptado de González-Partida et al. (2006) y de Camprubí et al. (2006b). Clave: Tm = temperatura de fusión.

Ahora bien, es preciso recordar que la conjunción de elementos de discusión favorables a la presencia de mineralizaciones económicas no implica necesariamente que éstas se encuentren presentes, pues no pueden producirse concentraciones importantes a partir de fluidos que no transportan suficientes metales en solución (Camprubí y Albinson, 2007).

\subsection{Zonaciones composicionales}

El establecimiento de zonaciones composicionales (salinidad, cantidad relativa de $\mathrm{CaCl}_{2}$ o $\mathrm{KCl}$, de $\mathrm{CO}_{2}$ o $\mathrm{CH}_{4}$, etc.) a nivel de yacimiento o de distrito minero es útil para determinar patrones de mezcla de fluidos cualesquiera. Tal es el caso de la mezcla de fluidos hipogénicos ascendentes, de cualquier origen, con (1) aguas meteóricas relativamente frías o (2) aguas calentadas por vapor (exsuelto en profundidad por ebullición) en la parte superior de los depósitos epitermales (Figura 9A) o (3) de la mezcla profunda de fluidos magmáticos con aguas meteóricas de circulación profunda en pórfidos metalíferos y skarns. Así, determinaciones de este tipo contribuyen a determinar o corroborar la existencia de determinados procesos geológicos, y ello conlleva la definición de criterios de exploración. Ahora bien, no es posible determinar con total seguridad el origen o el historial de interacción de los fluidos geológicos sólo con el uso de la microtermometría de inclusiones fluidas. Para ello se precisan criterios geológicos regionales sólidamente establecidos y el uso de técnicas analíticas adicionales, como la geoquímica de isótopos estables de $\mathrm{O}, \mathrm{H}, \mathrm{C}$ (y la modelación de la interacción agua/roca que ello permite) o de gases nobles, la espectrometría de masas cuadrupolar (QMS), etc. Sin embargo, el poder acotar con facilidad la composición en solutos de los fluidos geológicos constituye la pauta de inicio para ello y permite enunciar patrones de interacción concretos.

El valor práctico que posee el establecimiento de zonaciones composicionales a lo largo o a través de un 
cuerpo mineralizado (Figura 8 y 9A) lo constituye el hecho que a éstas puedan asociarse fenómenos o características geológicas favorables al hallazgo de nuevos recursos. Ello dependerá del tipo de yacimiento en estudio o exploración y de las condiciones fisicoquímicas en que se produce la precipitación mineral en éste, y no siempre puede generalizarse con facilidad. Así, en la parte superior de los depósitos epitermales es donde puede producirse un mayor grado de mezcla entre fluidos ascendentes y descendentes (aunque durante ciertas pausas entre grandes etapas de ascenso de fluidos hipogénicos y de mineralización, o durante la terminación de los sistemas hidrotermales, los fluidos descendentes pueden alcanzar profundidades considerables). Sin embargo, las porciones superiores de estos depósitos son las que están más expuestas a la erosión, a no ser que se produzca un rápido sepultamiento de éstos, lo que conlleva que los patrones de mezcla de fluidos sean difícilmente determinables en toda su extensión. De forma análoga, se encontrarán las salinidades más altas hacia fuentes magmáticas o en profundidad. Si es posible asociar una u otra característica con la presencia de mineralizaciones económicas, el establecimiento de zonaciones composicionales constituirá una importante guía de exploración.

En el caso de los depósitos metalíferos ( $\mathrm{Cu}-\mathrm{Au}-\mathrm{Mo})$ en pórfidos, el enfriamiento y dilución de los fluidos magmáticos, a partir de su interacción con fluidos más fríos externos al sistema magmático, constituyen mecanismos plausibles para desencadenar la precipitación mineral (Wilkinson, 2001). Los fluidos magmáticos se reconocen generalmente como (1) salmueras de alta salinidad $(\leq \sim 70$ wt.\% $\mathrm{NaCl}$ equiv.), reconocibles en este tipo de depósitos como inclusiones fluidas con cristales hijos de mineralogía diversa, o bien (2) vapores que, al condensar, pueden originar líquidos poco salinos. Bajo esa premisa, es posible modelizar la interacción entre un fluido magmático u otro y fluidos externos más fríos que, a pesar de que pueden presentar salinidades variables, éstas serán generalmente más bajas (y con una composición en solutos menos compleja) que las de los fluidos magmáticos ascendentes. Ello es debido a que esos fluidos más fríos son típicamente de origen meteórico. De esta forma, la identificación de patrones de variación en la salinidad y la composición de los solutos en inclusiones fluidas permitiría la delimitación de (1) áreas dominadas por fluidos magmáticos o meteóricos y (2) de áreas en que se produjo una interacción efectiva entre ambos tipos de fluidos. Tanto si se identifica un caso como el otro pueden establecerse criterios para guiar la exploración hacia zonas teóricamente favorables para el hallazgo de concentraciones minerales económicas. Sin embargo, hay que tener en cuenta que ello sólo es posible bajo la premisa general de que las mineralizaciones fueron originadas por la interacción entre fluidos de diferente origen, lo que no siempre es plenamente argumentable (pueden estar involucrados diferentes mecanismos de precipitación mineral en una misma asociación mineral). Además, en el caso particular de los pórfidos molibdeníferos de tipo Climax, las salinidades de los fluidos suelen ser más bajas que en otros pórfidos metalíferos (Cline y Vanko, 1995), por lo que la dilución de los fluidos magmáticos ascendentes puede no ser fácilmente modelable.

4.6. Zonación térmica y composicional a escala de provincia metalogenética

Algunas tipologías de yacimientos describen zonaciones (mineralógicas, térmicas, y en composición de fluidos) a nivel regional. En ellas la microtermometría de inclusiones fluidas provee información también a escala de provincia metalogenética. Tal es el caso de tipologías relacionadas con la evolución y circulación de salmueras de cuenca (ver Figura 8 en González-Partida et al., 2008b), como los depósitos tipo Mississippi Valley (MVT) y asociados, depósitos de $\mathrm{Cu}-\mathrm{Co}-\mathrm{U}$ en rocas clásticas (depósitos metalíferos asociados a red beds), así como reservorios de hidrocarburos (gas y petróleo).

La Provincia MVT del Noreste de México es un buen ejemplo de ello, con una clara zonación en la Cuenca de Sabinas de depósitos de celestina, fluorita (en los bordes sur y norte de la cuenca, respectivamente), barita, y Pb$\mathrm{Zn}$ (en el centro de la cuenca). Según este esquema, los depósitos formados a mayor profundidad en la cuenca, a mayores temperaturas $\left(<\sim 200^{\circ} \mathrm{C}\right)$ y a partir de salmueras más salinas ( $<\sim 22$ wt.\% $\mathrm{NaCl}$ equiv.) y ricas en $\mathrm{CaCl}_{2}$ son los depósitos de barita y $\mathrm{Pb}-\mathrm{Zn}$ (González-Sánchez et al., 2007). En el caso concreto de esta provincia metalogenética, el análisis microtermométrico de inclusiones fluidas, establecido de forma sistemática en toda la extensión de la provincia, contribuyó decisivamente a definir la tipología de los yacimientos en ésta. La definición de estos yacimientos como MVT y asociados constituye en sí misma una guía de exploración independientemente del tamaño de los yacimientos.

Igualmente, en cuencas petrolíferas puede determinarse la evolución en temperatura, presión y composición de salmueras acuosas e hidrocarburos. Ello, a través del análisis microtermométrico sistemático en (1) zonas productoras o de maduración inicial de hidrocarburos, (2) zonas de migración y (3) llenado de reservorios (Figura 8 en González-Partida et al., 2008b).

\section{Estimaciones de la presión de los fluidos y de la posición de paleoniveles freáticos}

En sistemas hidrotermales en ebullición o efervescencia bajo presión hidrostática es posible determinar la posición de la paleosuperficie durante el funcionamiento de éstos 0 , al menos, la posición del paleonivel freático. Ello es posible mediante las curvas de punto de ebullición del agua según la salinidad de los líquidos acuosos en el campo de la temperatura y la presión (o profundidad) de Haas (1971) y de las curvas de punto de efervescencia de $\mathrm{CO}_{2}$ de 
Henley et al. (1984). Estas estimaciones son especialmente útiles en depósitos epitermales (Albinson, 1988; Albinson et al., 2001; Camprubí et al., 2001b, 2006a). En principio, cualquier evidencia de ebullición en una posición concreta permite ubicar sobre la curva de ebullición del agua a la salinidad correspondiente el conjunto de datos de temperatura de homogeneización (por lo común representados mediante histogramas). Así, se posiciona la muestra en el punto de la curva que equivale a la temperatura de homogeneización y a la salinidad determinadas mediante microtermometría de inclusiones fluidas (bien sean como promedio de una asociación de inclusiones fluidas o varias de ellas, bien como datos de inclusiones individuales), de forma que la lectura de la presión y de la profundidad de formación es directa (Figura 10). Sin embargo, la profundidad obtenida debe entenderse como la distancia entre la muestra y el paleonivel freático, con lo que constituye una estimación mínima de la profundidad de formación. Puesto que la posición del paleonivel freático es cambiante según las condiciones hidrológicas de la región, el uso de las curvas de ebullición y efervescencia permite adicionalmente argumentar cambios en el paleogradiente hidráulico en el tiempo (elevación o depresión de las aguas freáticas), que a su vez pueden deberse a diferentes causas, entre ellas el clima. El volcanismo es uno de los factores principales a considerar, pues puede modificar drásticamente la posición de la paleosuperficie en ambos sentidos, por el colapso de edificios volcánicos o la rápida acumulación de material volcánico durante periodos eruptivos.

Tales reconstrucciones permiten guiar la exploración en profundidad en tipologías de yacimientos en que las franjas mineralizadas se distribuyen en rangos de profundidad concretos (como los depósitos epitermales). Asimismo, en yacimientos fallados pueden constituir un elemento adicional para estimar saltos de falla y, por tanto, localizar en profundidad recursos ocultos. Del mismo modo, también pueden condenar la exploración hacia zonas en que las mineralizaciones habrían sido erosionadas.

\section{Mecanismos de precipitación mineral}

Los mecanismos de precipitación mineral pueden ser de una naturaleza tan diversa como los propios yacimientos y en uno solo de ellos pueden haberse producido varios mecanismos, incluso al mismo tiempo. Entre ellos se cuentan ebullición, efervescencia, evaporación, sedimentación, cristalización, mezcla de fluidos, reacciones entre fluidos y rocas o entre rocas, enfriamiento conductivo y acción de microorganismos. Algunos de estos procesos pueden identificarse petrográfica y/o microtermométricamente.

Además de las evidencias mineralógicas y texturales (e. g. en yacimientos epitermales, presencia de calcita hojosa, de adularia pseudoromboédrica o pseudoacicular, de truscottita; ver Camprubí y Albinson, 2006), la ebullición puede ponerse igualmente de manifiesto mediante el estudio de inclusiones fluidas o a partir de las relaciones mo- lares entre los gases contenidos en ellas. Si en las zonas de ebullición se produce el atrapamiento de inclusiones fluidas, en éstas se presentará un amplio rango de variación de las relaciones líquido/vapor. Cabe destacar que, de hallarse sólo inclusiones muy ricas en vapor e inclusiones muy ricas en líquido, sin inclusiones con relaciones líquido/ vapor intermedias, es más posible que nos encontremos en una situación que refleja más bien un proceso de estrangulamiento de las inclusiones fluidas (también denominado encuellamiento, o necking). En tal caso es preferible optar por la cautela y no deducir que ello constituye evidencia de ebullición. La presencia de agrupaciones de inclusiones fluidas en que todas ellas son ricas en vapor, en cambio, constituye una mejor evidencia de ebullición. No siempre es posible encontrar inclusiones fluidas dentro de la propia zona de ebullición, pero pueden hallarse formas indirectas para evidenciar la existencia de este mecanismo de precipitación. Puesto que durante la ebullición se produce la separación de una fase vapor (hacia la cual se fraccionarán mayoritariamente los componentes más volátiles) y de una fase líquida (que adquirirá una mayor salinidad que el fluido que entró en ebullición), cabe esperar encontrar inclusiones ricas en líquido con salinidades relativamente más altas que las inclusiones anteriores, representativas de los fluidos anteriores a la ebullición. Ello, sin que haya necesariamente variaciones significativas en las temperaturas de homogeneización de unas y otras. La existencia de una sucesión como la expuesta también puede implicar la entrada de fluidos en un nuevo pulso hidrotermal sin relación alguna con la ebullición, con lo que el estudio de inclusiones fluidas tiene que apoyarse generalmente en otros métodos de análisis geoquímico (estudio de isótopos estables, geoquímica de gases, etc.). La diferencia entre ambos casos es que una ebullición puede generar "salmueras falsas", es decir, fluidos con salinidades anómalamente elevadas respecto al conjunto de datos al que pertenecen que no son representativas de la composición de fluido hipogénico alguno. Tales salmueras se habrían originado por ebullición continua "hacia el secado" de la salmuera original (Simmons y Browne, 1997). Por tanto, las salmueras debidas a ebullición no deben confundirse con fluidos ascendentes hipogénicos cuyas salinidades son originalmente elevadas o "salmueras verdaderas" (Figura 2). En la Figura 3 se ejemplifica el caso de una asociación de inclusiones fluidas con salinidades netamente mayores al resto de asociaciones de la misma etapa de mineralización. Si bien ello permite sospechar que dichos datos corresponden a una "falsa salmuera", la distribución de los datos ( $\sin$ datos con salinidades intermedias entre dichas asociaciones de inclusiones fluidas) sugiere que la posibilidad más razonable es que dicha salmuera sea "verdadera".

La existencia de ebullición, enfriamiento conductivo y diferentes tipos de mezcla de fluidos pueden ponerse de manifiesto a través del comportamiento de la distribución de los datos microtermométricos obtenidos de una asociación mineral concreta (Figura 3, 4 y 5). Sin embargo, la 
ETAPA I

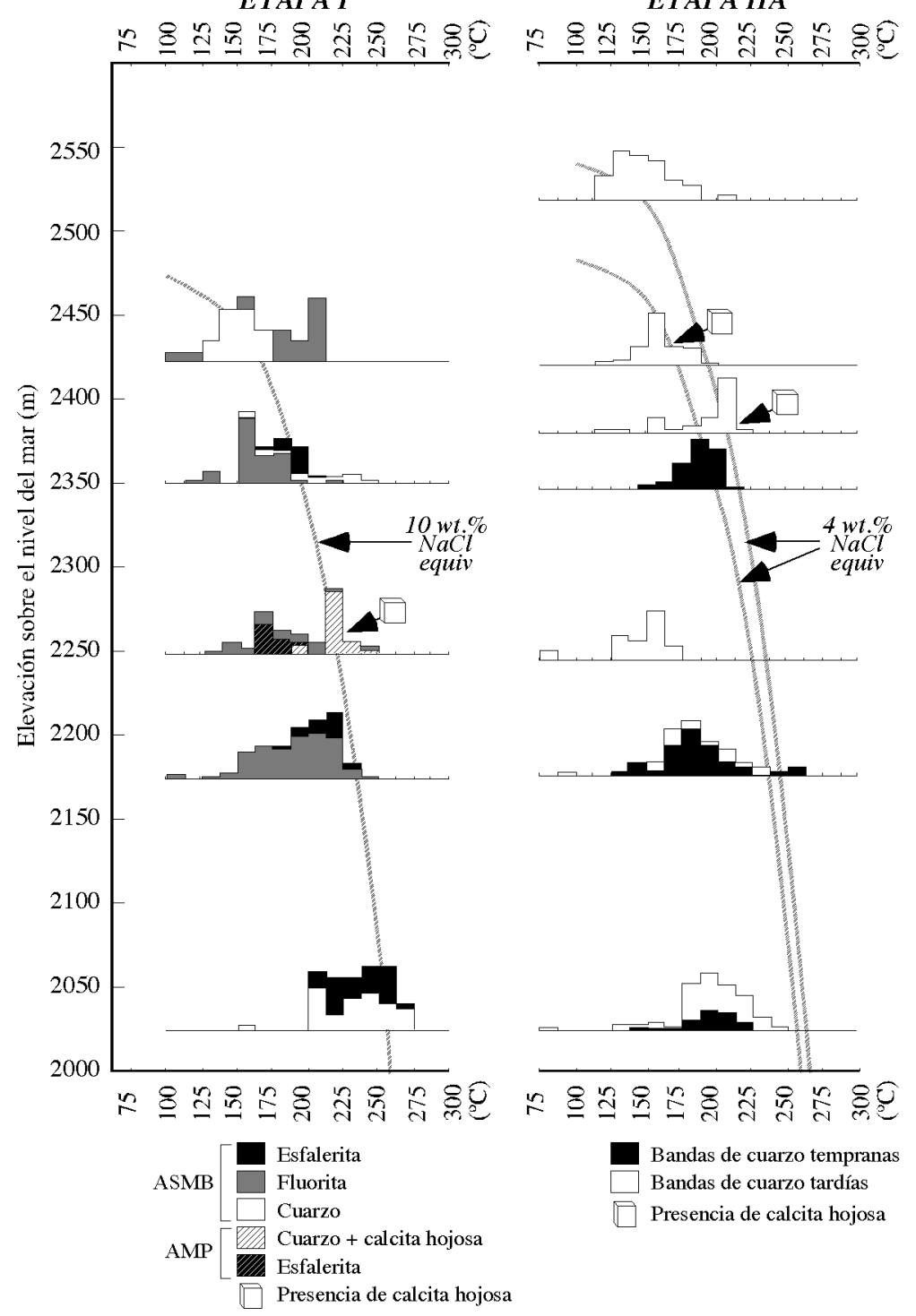

ETAPA IIB

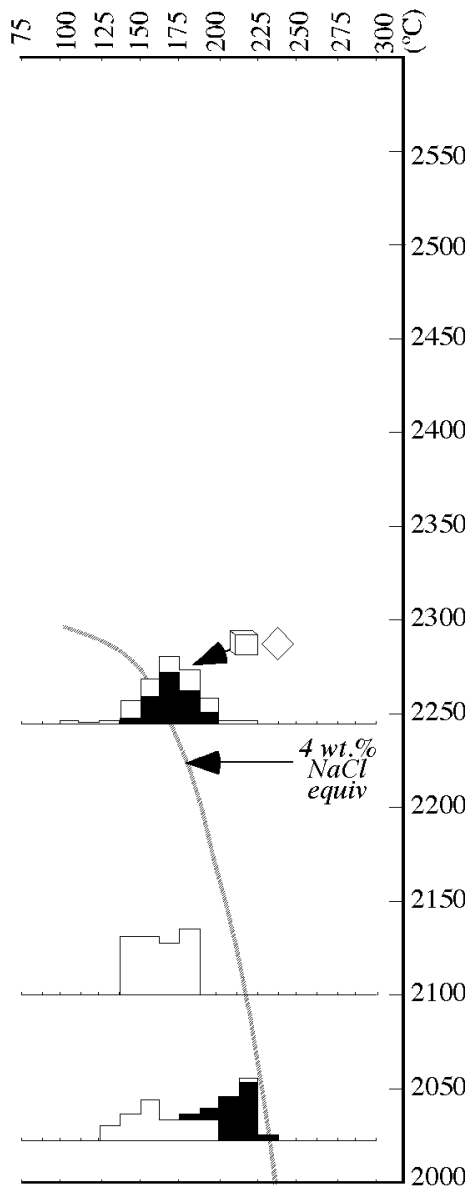

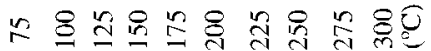

Bandas de cuarzo tempranas Bandas de cuarzo tardías

$\square$ Presencia de calcita hojosa Presencia de adularia

Figura 10. Histogramas de frecuencia de las temperaturas de homogeneización en inclusiones fluidas del yacimiento epitermal La Guitarra (distrito de Temascaltepec, Estado de México), mostrando las variaciones de temperatura con la profundidad de las etapas de mineralización I (mineralización de sulfuración intermedia), IIA y IIB (mineralizaciones de sulfuración baja). Se muestran las curvas del punto de ebullición bajo presión hidrostática de Haas (1971), para 10 wt.\% $\mathrm{NaCl}$ equiv. en la etapa I (referida a los datos en cuarzo de la AMP), 4 wt.\% NaCl equiv. en las etapas IIA y IIB. ASMB = asociación de sulfuros de metales básicos, AMP = asociación de minerales de plata. Adaptado de Camprubí et al. (2001b).

interpretación de este tipo de procesos obliga a poseer un alto grado de control petrográfico. En resumen, la distribución de los datos microtermométricos en un diagrama de temperatura de homogeneización o de atrapamiento contra salinidad puede ayudar a caracterizar procesos geológicos. Algunos de ellos pueden haber conllevado la precipitación mineral (Figura 4), con lo que la identificación de tendencias características en el comportamiento de los datos es esencial. Estos procesos y tendencias son los siguientes:

Ebullición, o separación de vapor de un fluido acuoso (Figura 3 y 4). Como ya se expuso anteriormente, una ebullición genera salmueras progresivamente más salinas y densas por la fuerte partición de las sales hacia las fases líquidas. Como resultado de su expansión adiabática, estas salmueras pueden también experimentar un cierto enfriamiento (Wilkinson, 2001). Sin embargo, la ebullición también genera vapores de mayor temperatura que el fluido pre-ebullición, que eventualmente pueden condensarse y generar líquidos de muy baja salinidad y alta acidez, independientemente del quimismo del fluido pre-ebullición. Los mismos efectos pueden registrarse tras la efervescencia de los fluidos mineralizantes. Este mecanismo de deposición es especialmente eficiente en depósitos epitermales, skarns y pórfidos metalíferos.

Efervescencia, o separación de $\mathrm{CO}_{2}, \mathrm{CH}_{4}$ u otros gases a partir de un fluido acuoso. Si la presencia de dichos com- 
puestos (en fluidos pre-efervescencia o en condensados someros post-efervescencia) no puede ser determinada ópticamente, su presencia puede detectarse mediante la fusión de clatratos o mediante el uso de técnicas analíticas adicionales (e. g., microespectroscopía Raman). Este mecanismo de deposición es común en yacimientos tipo MVT y asociados.

Mezcla de fluidos. Este proceso precisa de una identificación correcta de la temperatura y salinidad de los miembros extremos que lo experimentan. La trayectoria de datos reveladores de un proceso de este tipo depende de las características de dichos miembros, y pueden llegarse a confundir con las trayectorias debidas a otros procesos. En algunos casos (e. g. depósitos tipo MVT) se ha determinado que el azufre y los metales necesarios para formar cuerpos de sulfuros no pueden ser transportados en el mismo fluido, por lo que la precipitación mineral se produce por mezcla de dos fluidos (Sverjensky, 1984). Los casos de mezcla más comunes en la naturaleza son:

Dilución (Figura 3, 4 y 5). Este es un caso especialmente abundante en la naturaleza, en que aguas meteóricas relativamente frías y poco salinas (aunque hayan experimentado un cierto grado de interacción cortical) pueden mezclarse con salmueras acuosas hipogénicas de origen profundo, más calientes y salinas. Estas últimas pueden ser aguas meteóricas con un grado de interacción cortical profundo y muy prolongado. Aunque no siempre es fácil explicar este tipo de mezclas por las diferencias en densidad de los fluidos que intervienen en ellas, la turbulencia de los fluidos ascendentes es permisiva para una mezcla eficiente. Se reconoce cuando el comportamiento de los datos microtermométricos describe una marcada tendencia a la disminución de temperatura y salinidad al mismo tiempo. Este mecanismo de deposición es común en yacimientos tipo MVT someros y en muchas otras tipologías, incluyendo depósitos de origen magmático-hidrotermal.

Mezcla isotérmica. Este proceso se produce generalmente entre fluidos con un remarcable contraste en la salinidad pero a temperaturas muy parecidas, debido a que ambos se encuentran en un régimen geotérmico análogo y han adquirido el mismo grado de equilibrio térmico con el medio. Se caracteriza por presentar conjuntos de datos con variaciones apreciables en salinidad aunque no de temperatura.

Enfriamiento conductivo (Figura 3). Se produce cuando un fluido pierde calor por conducción a través de las rocas circundantes sin que varíe significativamente la composición química del fluido. Se caracteriza por presentar conjuntos de datos con variaciones apreciables en temperatura, hacia temperaturas más bajas con el tiempo, aunque no de salinidad. Como mecanismo de precipitación es común en depósitos formados en la porción superior de la corteza, pues en las porciones más profundas de la misma no existen gradientes térmicos lo suficientemente extremos como para inducir precipitación mineral (Wilkinson, 2001).
Disolución de evaporitas. Los fluidos de cualquier origen que atraviesen series evaporíticas registrarán salinidades anómalamente altas, aunque no todos los fluidos asociados a un sistema hidrotermal deben experimentar el mismo grado de interacción con las evaporitas. Por ello, las salinidades de los fluidos pueden ser muy variables sin razón aparente. La posibilidad de este tipo de interacción puede deducirse a partir de evidencias geológicas regionales. Teniendo en cuenta la importancia del $\mathrm{Cl}$ como agente de transporte de metales en forma de ión complejante en condiciones geológicas muy diversas, la determinación del posible origen del mismo es un factor crucial para comprender el origen de las soluciones mineralizantes.

Régimen hidrotermal pulsátil (Figura 2). En sistemas pulsátiles, pueden identificarse pulsos discretos a diferentes escalas de observación, aunque no siempre sean claramente identificables. La variable más fiable en este aspecto es la salinidad. De registrar la incorporación pulsátil de fluidos, generalmente a partir de un reservorio profundo, lo más común es observar que asociaciones de inclusiones fluidas sucesivas muestren algún tipo de tendencia de disminución o aumento en la salinidad, independientemente de la temperatura de homogeneización.

Estrangulamiento (encuellamiento) de inclusiones fluidas. Este cambio post-atrapamiento, asociado a la recristalización de los minerales que contienen inclusiones fluidas, induce la partición de las inclusiones. Si ésta se produce cuando alguna fase se ha separado en el interior de la inclusión, pueden generarse nuevas inclusiones fluidas con grados de relleno muy diversos. Ello produce temperaturas de homogeneización variables pero salinidades relativamente homogéneas. En consecuencia, los datos microtermométricos derivados de este proceso pueden presentar distribuciones parecidas a las del enfriamiento conductivo o de la ebullición. La diferencia es que, en el caso de haberse producido un estrangulamiento, tal comportamiento de los datos se produce a nivel de la misma asociación de inclusiones fluidas, mientras que los demás procesos registran ese comportamiento a través de asociaciones de inclusiones fluidas diferentes. Este proceso sólo afecta a las inclusiones fluidas, pero no tiene que ver con procesos de precipitación mineral o de interacción agua/roca.

Otros cambios post-atrapamiento: decrepitación y relleno y dilatación. El efecto de la decrepitación y relleno es parecido al de la dilución, mientras que el de la dilatación es parecido al del enfriamiento conductivo. En el caso de producirse ambos cambios combinados, lo cual es común, la distribución de los datos microtermométricos "apunta" hacia la salinidad y temperatura del fluido original (Goldstein y Reynolds, 1994).

\section{Agradecimientos}

La elaboración de diversas partes del presente trabajo se financió mediante los proyectos de investigación $\mathrm{J}$ 32506-T y 46473 de CONACYT e IN103807 de PAPIIT. 
El autor agradece especialmente las revisiones críticas de Tawn Albinson, Pura Alfonso y Joaquín Proenza, que ayudaron a mejorar sensiblemente el presente trabajo. Igualmente, agradezco la invitación a participar, mediante una contribución germinal de este trabajo, en la primera edición del Taller Iberoamericano de Recursos Minerales y Apoyo a la Pequeña Minería a través de Joaquín Proenza, Jesús Blanco y Joan-Carles Melgarejo.

\section{Bibliografía}

Albinson, T.F., 1988, Geologic reconstruction of paleosurfaces in the Sombrerete, Colorada, and Fresnillo districts, Zacatecas State, Mexico: Economic Geology, 83, 1647-1667.

Albinson, T., 1995, Bosquejo de evolución estructural e hidrotermal del distrito de Zacatecas, en Trabajos Técnicos XXI Convención Nacional de la AIMMGM: Acapulco, Guerrero, México, Asociación de Ingenieros de Minas, Metalurgistas y Geólogos de México, 143-170.

Albinson, T., Rubio, M.A., 2001, Mineralogic and thermal structure of the Zuloaga Vein, San Martin de Bolaños District, Jalisco, Mexico, in Albinson, T., Nelson, C.E. (eds.), New mines and discoveries in Mexico and Central America: Society of Economic Geologists Special Publication Series, 8, 115-132.

Albinson, T., Norman, D.I., Cole, D., Chomiak, B.A., 2001, Controls on formation of low-sulfidation epithermal deposits in Mexico: constraints from fluid inclusion and stable isotope data, in: Albinson, T., Nelson, C.E. (eds.), New mines and discoveries in Mexico and Central America: Society of Economic Geologists Special Publication $8,1-32$.

Becker, S.P., Fall, A., Bodnar, R.J., 2008, Synthetic fluid inclusions. XVII. PVTX properties of high salinity $\mathrm{H}_{2} \mathrm{O}-\mathrm{NaCl}$ solutions $(>30$ wt $\% \mathrm{NaCl}$ ): application to fluid inclusions that homogenize by halite disappearance from porphyry copper and other hydrothermal ore deposits: Economic Geology, 103, 539-554.

Camprubí, A., Albinson, T., 2006, Depósitos epitermales en México: actualización de su conocimiento y reclasificación empírica: Boletín de la Sociedad Geológica Mexicana, 58, 27-81.

Camprubí, A., Albinson, T., 2007, Epithermal deposits in México - an update of current knowledge, and an empirical reclassification, in Alaniz-Álvarez, S.A., Nieto-Samaniego, A.F. (eds.), Geology of México: Celebrating the Centenary of the Geological Society of México: Geological Society of America Special Paper, 422, $377-415$.

Camprubí, A., Canals, À., Cardellach, E., Prol-Ledesma, R.M., Rivera, R., 2001a, The La Guitarra Ag-Au low sulfidation epithermal deposit, Temascaltepec district, Mexico: vein structure, mineralogy, and sulfide-sulfosalt chemistry, in Albinson, T., Nelson, C.E. (eds.), New mines and discoveries in Mexico and Central America, Society of Economic Geologists Special Publication, 8, 133-158.

Camprubí, A., Cardellach, E., Canals, À., Lucchini, R., 2001b, The La Guitarra Ag-Au low sulfidation epithermal deposit, Temascaltepec district, Mexico: fluid inclusion and stable isotope data, in Albinson, T., Nelson, C.E. (eds.), New mines and discoveries in Mexico and Central America, Society of Economic Geologists Special Publication, 8, 159-185.

Camprubí, A., González-Partida, E., Iriondo, A., Levresse, G., 2006a, Mineralogy, fluid characteristics and depositional environment of the Paleocene low-sulfidation epithermal Au-Ag deposits of the El Barqueño district, Jalisco, Mexico: Economic Geology, 101, 235-247.

Camprubí, A., González-Partida, E., Torres-Tafolla, E., 2006b, Fluid inclusion and stable isotope study of the Cobre - Babilonia polymetallic epithermal vein system, Taxco district, Guerrero, Mexico: Journal of Geochemical Exploration, 89, 33-38.

Canet, C., Camprubí, A., González-Partida, E., Linares, C., Alfonso, P., Piñeiro-Fernández, F., Prol-Ledesma, R.M., 2009, Mineral assem- blages of the Francisco I. Madero $\mathrm{Zn}-\mathrm{Cu}-\mathrm{Pb}-(\mathrm{Ag})$ deposit, Zacatecas, Mexico: implications for ore deposit genesis: Ore Geology Reviews, 35, 423-435.

Cline, J.S., Vanko, D.A., 1995, Magmatically generated saline brines related to molybdenum at Questa, New Mexico, USA, in Thompson, J.F.H. (ed.), Magmas, fluids, and ore deposits: Mineralogical Association of Canada Short Course Series, 23, 153-174.

Crawford, M.L., 1981, Phase equilibria in aqueous fluid inclusions, in Hollister, L.S., Crawford, M.L. (eds.), Fluid inclusions: Applications to Petrology: Calgary, Mineralogical Association of Canada Short Course Handbook 6, 75-100.

Dong, G., Morrison, G., Jaireth, S., 1995, Quartz textures in epithermal veins, Queensland; classification, origin, and implication: Economic Geology, 90, 1841-1856.

Fournier, R.O., 1999, Hydrothermal processes related to movement of fluid from plastic into brittle rock in the magmatic-epithermal environment: Economic Geology, 94, 1193-1211.

Garza-González, C., Camprubí, A., González-Partida, E., Arriaga-García, G., Rosique-Naranjo, F., 2006, Hydrothermal alteration and fluid inclusion study of the Lower Cretaceous porphyry $\mathrm{Cu}-\mathrm{Au}$ deposit of Tiámaro, Michoacán, Mexico: Journal of Geochemical Exploration, 89, 124-128.

Goldstein, R.H., Reynolds, T.J., 1994, Systematics of fluid inclusions in diagenetic minerals: Tulsa, EUA, SEPM Short Course 31, 199 p.

González-Partida, E., Camprubí, A., 2006, Evolution of mineralizing fluids in the $\mathrm{Zn}-\mathrm{Pb}-\mathrm{Cu}(-\mathrm{Ag} \pm \mathrm{Au})$ skarn and epithermal deposits of the world-class San Martín district, Zacatecas, Mexico: Journal of Geochemical Exploration, 89, 138-142.

González-Partida, E., Camprubí, A., Levresse, G., Tritlla, J., CarrilloChávez, A., 2004, Fluidos asociados al skarn Au(-Cu) de La Joya, distrito de Mezcala, Guerrero, México: implicaciones regionales para depósitos formados a partir de rocas calcoalcalinas vs. adakíticas: Revista Mexicana de Ciencias Geológicas, 21, 371-381.

González-Partida, E., Camprubí, A., González-Sánchez, F., SánchezTorres, J., 2006, Fluid inclusion study of the Plomositas - Los Arcos polymetallic epithermal vein tract, Plomosas district, Sinaloa, Mexico: Journal of Geochemical Exploration, 89, 143-148.

González-Partida, E., Camprubí, A., Canet, C., González-Sánchez, F., 2008a, Fisicoquímica de salmueras e hidrocarburos en cuencas petroleras y en depósitos minerales tipo Mississippi Valley y asociados. Parte I: temperatura, presión y composición de inclusiones fluidas: Boletín de la Sociedad Geológica Mexicana, 60, 11-22.

González-Partida, E., Camprubí, A., Canet, C., González-Sánchez, F., 2008b, Fisicoquímica de salmueras e hidrocarburos en cuencas petroleras y en depósitos minerales tipo Mississippi Valley y asociados. Parte II: ejemplos de la Cuenca de Sabinas y la Cuenca del Sureste, México: Boletín de la Sociedad Geológica Mexicana, 60, 23-42.

González-Sánchez, F., Puente-Solís, R., González-Partida, E., Camprubí, A., 2007, Estratigrafía del Noreste de México y su relación con los yacimientos estratoligados de fluorita, barita, celestina y $\mathrm{Zn}-\mathrm{Pb}$ : Boletín de la Sociedad Geológica Mexicana, 59 (1), 43-62.

Goodell, P.C., Petersen, U., 1974, Julcani mining district, Peru: a study of metal ratios: Economic Geology, 69, 347-361.

Haas, J.L., 1971, The effect of salinity on the maximum thermal gradient of a hydrothermal system at hydrostatic pressure: Economic Geology, 66, 940-946.

Harris, A.C., Golding, S.D., White, N.C., 2005, Bajo de la Alumbrera Copper-Gold Deposit: Stable Isotope Evidence for a PorphyryRelated Hydrothermal System Dominated by Magmatic Aqueous Fluids: Economic Geology, 100, 863-886.

Henley, R.W., Truesdell, A.H., Barton Jr., P.B., Whitney, J.A., 1984, Fluid-mineral equilibria in hydrothermal systems: Reviews in Economic Geology, 1, $267 \mathrm{p}$.

Reyes, A.G., 1990, Petrology of Philippine geothermal systems and the application of alteration mineralogy to their assessment: Journal of Volcanology and Geothermal Research, 43, 279-309.

Reyes, A.G., 1991, Mineralogy, distribution and origin of acid alteration in Philippine geothermal systems: Geological Survey of Japan Reports, 277, 59-65. 
Roedder, E., 1984, Fluid inclusions: Mineralogical Society of America Reviews in Mineralogy, 12, 644 p.

Sander, M.V., Black, J.E., 1988, Crystallization and recrystallization of growth-zoned vein quartz crystals from epithermal systems; implications for fluid inclusion studies: Economic Geology, 83, 1052-1060

Simmons, S.F., Browne, P.R.L., 1997, Saline fluid inclusions in sphalerite from the Broadlands-Ohaaki geothermal system; a coincidental trapping of fluids boiled towards dryness: Economic Geology, 92, 485-489.
Sverjensky, D.A., 1984, Oil field brines as ore-forming solutions: Economic Geology, 79, 23-37.

Van den Kerkhof, A.M., Hein, U.F., 2001, Fluid inclusion petrography: Lithos, 55, 27-47.

Wilkinson, J.J., 2001, Fluid inclusions in hydrothermal ore deposits: Lithos, 55, 229-272.

Wu, I., Petersen, U., 1977, Geochemistry of tetrahedrite and mineral zoning at Casapalca, Peru: Economic Geology, 72, 993-1016.

Recibido: 02/06/2008

Recibido corregido: 06/09/2008

Aceptado: 02/10/2008 